Supplemental Information

\title{
Discovery and Optimization of Triazine Nitrile Inhibitors of Toxoplasma gondii Cathepsin L for the Potential Treatment of Chronic Toxoplasmosis in the CNS
}

Jeffery D. Zwicker ${ }^{\dagger, \neq}$, David Smith ${ }^{\ddagger}$, Alfredo J. Guerra ${ }^{\ddagger}$, Jacob R. Hitchens ${ }^{\dagger \neq}$, Nicole Haug $\$$, Steve Vander Roest $^{\#}$, Pil Lee ${ }^{\dagger,}$, Bo Wen $\$$, Duxin Sun $\$$, Lu Wang§, Richard F. Keep ${ }^{+}$, Jianming Xiang ${ }^{+}$, Vern B. Carruthers $\$$, and Scott D. Larsen*广, $\neq$

†Vahlteich Medicinal Chemistry Core, College of Pharmacy, University of Michigan, Ann Arbor, Michigan 48109, United States

$\ddagger$ Department of Medicinal Chemistry, University of Michigan, Ann Arbor, Michigan 48109, United States Department of Microbiology and Immunology, University of Michigan, Ann Arbor, Michigan 48109, United States

\#Center for Chemical Genomics, Life Sciences Institute, Ann Arbor, MI 48109, United States

\$Pharmacokinetics Core, Department of Pharmaceutical Sciences, University of Michigan, Ann Arbor, Michigan 48109, United States

${ }^{+}$Department of Neurosurgery, University of Michigan, Ann Arbor, Michigan 48109, United States

\section{Chemistry Experimental:}

\section{General Procedure A1: Reductive Amination}

To a dry round bottom flask with DCM was added arylaldehyde (1 eq), followed by the addition of the desired primary amine (1 eq), final reaction concentration of 0.1-0.3 $\mathrm{mM}$. The vessel was then stirred under a nitrogen atmosphere at room temperature overnight. Sodium triacetoxyborohydride (3 eq) was then added and reaction was stirred at room temperature for 1-3 hr. The reaction was quenched slowly with water then poured into water and extracted 3x with DCM. The combined organic layer was dried over sodium sulfate and concentrated in-vacuo. The crude product was further purified by column chromatography $(0-10 \% \mathrm{MeOH}$ in DCM $+0.1 \%$ TEA) to afford the desired secondary amine. 


\section{General Procedure A2: $S_{\mathrm{N}} \mathrm{Ar}$}

Under a dry nitrogen atmosphere, cyanuric chloride (1 eq) was added a roundbottom flask along with DCM (final conc. $=0.1-0.3 \mathrm{mM})$. The vessel was cooled to $-10^{\circ} \mathrm{C}($ Ice/Brine) and the desired secondary amine ( 1 eq) and DIPEA ( 1 eq) were then added. Reaction was stirred at $-10^{\circ} \mathrm{C}$ for $1 \mathrm{~h}$. The reaction was poured into water and extracted 3x with DCM. Combined organic layers were dried over $\mathrm{MgSO}_{4}$ and concentrated in vacuo. The crude product was further purified by column chromatography (0$100 \%$ EtOAc in Hexanes gradient) to afford the desired intermediate.

\section{General Procedure A3: $S_{N} A r$}

Under a dry nitrogen atmosphere, dichloro triazine intermediate $(1 \mathrm{eq})$ was added a roundbottom flask along with DCM to a final reaction concentration of $0.1 \mathrm{mM}$. The vessel was cooled to $-10^{\circ} \mathrm{C}$ (Ice/Brine) and DIPEA (1 eq) and morpholine (1 eq) were then added. Reaction was stirred and allowed to warm to room temperature over 6-12 $\mathrm{h}$. The reaction was poured into water and extracted $3 \mathrm{x}$ with DCM. Combined organic layers were dried over $\mathrm{MgSO}_{4}$ and concentrated in vacuo. The crude product was further purified by column chromatography (0-100\% EtOAc in Hexanes gradient) to afford the desired intermediate.

\section{General Procedure A4: Cyanation}

A dry pressure vessel was charged with the appropriate triazinyl chloride (1.0 eq), and suspended in DMSO/ $\mathrm{H}_{2} \mathrm{O}$ 9:1. KCN (1.1 eq.) and 1,4-diazabicyclo[2.2.2] octane (DABCO, 2.0 eq.) were added and the reaction was heated to $80^{\circ} \mathrm{C}$. Reaction was left to stir at this temperature until LC/MS showed completion of the reaction (6-12 h). Reaction was cooled to room temperature, diluted with EtOAc, and washed thoroughly with brine (3-5 x). The organic layer was separated, dried over $\mathrm{MgSO}_{4}$, filtered, and evaporated. The crude product was further purified by column chromatography $(0-100 \%$ EtOAc in Hexanes gradient) to afford the desired product. 


\section{General Procedure B1: One-pot double $S_{N} A r$}

Under a dry nitrogen atmosphere, cyanuric chloride ( 1 eq) was added a roundbottom flask along with DCM (final conc. $=0.1-0.3 \mathrm{mM})$. The vessel was cooled to $-10^{\circ} \mathrm{C}($ Ice/Brine $)$ and DIPEA $(1 \mathrm{eq})$ followed by the desired primary amine (1eq) were then added. Reaction was stirred at for 1-3 hr, until TLC/HPLC indicated substitution was complete. Next, DIPEA (1 eq) was added followed by morpholine (1 eq) and reaction was allowed to stir and warm to rt over 4-12h until TLC/HPLC indicated substitution was complete. The reaction was poured into water and extracted 3x with DCM. Combined organic layers were dried over $\mathrm{MgSO}_{4}$ and concentrated in vacuo. The crude product was further purified by column chromatography (0-100\% EtOAc in Hexanes gradient) to afford the desired intermediate.

\section{General Procedure B2: Cyanation}

A dry pressure vessel was charged with the appropriate triazinyl chloride (1.0 eq), and suspended in $\mathrm{DMSO} / \mathrm{H}_{2} \mathrm{O}$ 9:1. KCN (1.1 eq) and 1,4-diazabicyclo[2.2.2] octane (DABCO, 2.0 eq) were added and the reaction was heated to $80^{\circ} \mathrm{C}$. Reaction was left to stir at this temperature until LC/MS showed completion of the reaction (6-12 h). Reaction was cooled to room temperature, diluted with EtOAc and washed thoroughly with brine (3-5 x). The organic layer was separated, dried over $\mathrm{MgSO}_{4}$, filtered, and evaporated. The crude product was further purified by column chromatography $(0-100 \%$ EtOAc in Hexanes gradient) to afford the desired secondary amine.

\section{General Procedure B3: Alkylation with $\mathrm{NaH}$ and aryl/alkyl X/Ms/Ts}

The triazinyl-nitrile ( 1 eq) was dissolved in DMF and cooled to $-10^{\circ} \mathrm{C}$ (Ice/Brine bath). $60 \% \mathrm{NaH}$ in mineral oil (1-1.5 eq) was added and reaction was stirred for $30 \mathrm{~min}$. The appropriate alkyl or aryl $\mathrm{Br} / \mathrm{Cl} / \mathrm{Ms} / \mathrm{Ts}$ (1-2 eq) was then added. The reaction was allowed to slowly warm to room temperature and left to stir (2-12 hr) until HPLC indicated reaction was complete. The solution was poured into EtOAc, 
washed $3 \mathrm{x}$ with brine, dried over $\mathrm{NaSO}_{4}$, and concentrated. Crude residue was purified by flash chromatography EtOAc:Hexanes 0-100\% gradient.

\section{General Procedure B4: Boc-Deprotection}

The Boc-protected amine (1 eq) was dissolved into a 1:2 mixture of TFA:DCM ( 0.1-0.3 mM) and allowed to stir at room temperature for $0.5-2 \mathrm{~h}$, until HPLC or TLC indicated reaction was complete. Solvent was removed in vacuo and compound was purified by reverse phase chromatography $(\mathrm{C} 18,10-$ $100 \% \mathrm{ACN}$ in water $+0.1 \%$ TFA gradient) and concentrated to afford desired product as the TFA salt. All final compounds exist as rotamers on the NMR timescale.

\section{N-(4-Fluorobenzyl)-3-methylbutan-1-amine (Scheme 1, Int-2a)}

General Procedure A1 from 4-fluorobenzaldehyde (0.5 ml, $4.66 \mathrm{mmol}), 3$-methylbutan-1-amine (0.541 $\mathrm{ml}, 4.66 \mathrm{mmol})$, and sodium triacetoxyborohydride (2470 mg, $11.65 \mathrm{mmol}$ ) gave N-(4-fluorobenzyl)-3methylbutan-1-amine (870 mg, $4.46 \mathrm{mmol}, 96 \%$ yield).; ${ }^{1} \mathrm{H} \mathrm{NMR}\left(\mathrm{CDCl}_{3}, 500 \mathrm{MHz}\right) \delta 7.27$ (dd, $J=$ 5.62, 8.07 Hz, 2H), 6.99 (t, $J=8.56 \mathrm{~Hz}, 2 \mathrm{H}), 3.75(\mathrm{~s}, 2 \mathrm{H}), 2.62(\mathrm{t}, J=7.34 \mathrm{~Hz}, 2 \mathrm{H}), 1.56-1.69$ (m, 1H), $1.34-1.45(\mathrm{~m}, 2 \mathrm{H}), 0.89(\mathrm{~d}, J=6.36 \mathrm{~Hz}, 6 \mathrm{H})$

\section{4,6-Dichloro-N-(4-fluorobenzyl)-N-isopentyl-1,3,5-triazin-2-amine (Scheme 1, Int-3a)}

General Procedure A2 from 2,4,6-trichloro-1,3,5-triazine (142 mg, $0.768 \mathrm{mmol}$ ), N-(4-fluorobenzyl)-3methylbutan-1-amine (150 mg, $0.768 \mathrm{mmol})$, and DIPEA $(0.134 \mathrm{ml}, 0.768 \mathrm{mmol})$ gave 4,6-dichloro-N(4-fluorobenzyl)-N-isopentyl-1,3,5-triazin-2-amine (243 mg, $0.708 \mathrm{mmol}, 92 \%$ yield).; ${ }^{1} \mathrm{H} \mathrm{NMR}\left(\mathrm{CDCl}_{3}\right.$, $500 \mathrm{MHz}) \delta 7.23(\mathrm{dd}, J=5.38,8.31 \mathrm{~Hz}, 2 \mathrm{H}), 7.03(\mathrm{t}, J=8.56 \mathrm{~Hz}, 2 \mathrm{H}), 4.79(\mathrm{~s}, 2 \mathrm{H}), 3.51-3.56(\mathrm{~m}, 2 \mathrm{H})$, $1.49-1.61(\mathrm{~m}, 1 \mathrm{H}), 1.40-1.48(\mathrm{~m}, 2 \mathrm{H}), 0.92(\mathrm{~d}, J=6.36 \mathrm{~Hz}, 6 \mathrm{H})$

\section{4-Chloro-N-(4-fluorobenzyl)-N-isopentyl-6-morpholino-1,3,5-triazin-2-amine (Scheme 1, Int-4a)}


General procedure A3 from 4,6-dichloro-N-(4-fluorobenzyl)-N-isopentyl-1,3,5-triazin-2-amine (243 mg, $0.708 \mathrm{mmol})$, morpholine $(0.062 \mathrm{ml}, 0.708 \mathrm{mmol})$, and DIPEA $(0.124 \mathrm{ml}, 0.708 \mathrm{mmol})$ gave 4-chloro-N(4-fluorobenzyl)-N-isopentyl-6-morpholino-1,3,5-triazin-2-amine (205 mg, $0.520 \mathrm{mmol}, 73.5 \%$ yield).

Product could be recrystallized from hot EtOAc:Hexanes (2:1).; ${ }^{1} \mathrm{H} \mathrm{NMR}\left(\mathrm{CDCl}_{3}, 500 \mathrm{MHz}\right) \delta 7.15-$ $7.25(\mathrm{~m}, 2 \mathrm{H}), 6.99(\mathrm{t}, J=8.56 \mathrm{~Hz}, 2 \mathrm{H}), 4.74(\mathrm{~s}, 2 \mathrm{H}), 3.73-3.88(\mathrm{~m}, 4 \mathrm{H}), 3.60-3.73(\mathrm{~m}, 5 \mathrm{H}), 3.49-3.56$ $(\mathrm{m}, 1 \mathrm{H}), 3.39-3.46(\mathrm{~m}, 1 \mathrm{H}), 1.47-1.61(\mathrm{~m}, 1 \mathrm{H}), 1.39-1.46(\mathrm{~m}, 2 \mathrm{H}), 0.89(\mathrm{t}, J=6.85 \mathrm{~Hz}, 6 \mathrm{H})$

\section{4-((4-Fluorobenzyl)(isopentyl)amino)-6-morpholino-1,3,5-triazine-2-carbonitrile (4)}

General Procedure A4 starting from 4-chloro-N-(4-fluorobenzyl)-N-isopentyl-6-morpholino-1,3,5-triazin2-amine (200 mg, $0.508 \mathrm{mmol})$ and DABCO (114 mg, 1.016 mmol) gave 4-((4-

fluorobenzyl)(isopentyl)amino)-6-morpholino-1,3,5-triazine-2-carbonitrile (136 mg, $0.354 \mathrm{mmol}, 69.7 \%$ yield); ${ }^{1} \mathrm{H}$ NMR $\left(\mathrm{CDCl}_{3}, 400 \mathrm{MHz}\right) \delta 7.13$ - $7.25(\mathrm{~m}, 2 \mathrm{H}), 7.00(\mathrm{t}, \mathrm{J}=8.22 \mathrm{~Hz}, 2 \mathrm{H}), 4.73$ (d, J = 19.17 Hz, 2H), 3.74 - 3.87 (m, 4H), 3.60 - $3.74(\mathrm{~m}, 6 \mathrm{H}), 3.52(\mathrm{t}, \mathrm{J}=7.43 \mathrm{~Hz}, 1 \mathrm{H}), 3.43(\mathrm{t}, \mathrm{J}=7.83 \mathrm{~Hz}, 1 \mathrm{H}), 1.48$ $1.60(\mathrm{~m}, 1 \mathrm{H}), 1.37-1.48(\mathrm{~m}, 2 \mathrm{H}), 0.90(\mathrm{t}, \mathrm{J}=5.87 \mathrm{~Hz}, 6 \mathrm{H}) ;{ }^{13} \mathrm{C} \mathrm{NMR}\left(\mathrm{DMSO}-d_{6}, 101 \mathrm{MHz}, 80^{\circ} \mathrm{C}\right) \delta$ $163.2,162.7,161.1,150.9,133.2,129.0,115.0,114.6,65.2,48.5,44.4,43.2,25.0,21.7$; HRMS-ESI (m/z): $[\mathrm{M}+\mathrm{H}]^{+}$calcd for $\mathrm{C}_{20} \mathrm{H}_{25} \mathrm{FN}_{6} \mathrm{O}, 385.2147$; found, 385.2144

\section{4-Chloro-N-(4-fluorobenzyl)-6-morpholino-1,3,5-triazin-2-amine (Scheme 2, Int-2b)}

General procedure B1 from 2,4,6-trichloro-1,3,5-triazine (1 g, 5.42 mmol), (4-fluorophenyl)methanamine (0.620 ml, $5.42 \mathrm{mmol})$, DIPEA (1.894 ml, $10.84 \mathrm{mmol})$, and morpholine (0.472 ml, $5.42 \mathrm{mmol})$ gave 4chloro-N-(4-fluorobenzyl)-6-morpholino-1,3,5-triazin-2-amine (1.07 g, $3.30 \mathrm{mmol}, 60.9 \%$ yield).

Product could be recrystallized from hot EtOAc:Hexanes (2:1).; ${ }^{1} \mathrm{H} \mathrm{NMR}\left(\mathrm{CDCl}_{3}, 500 \mathrm{MHz}\right) \delta 7.17$ $7.35(\mathrm{~m}, 2 \mathrm{H}), 6.89$ - $7.11(\mathrm{~m}, 2 \mathrm{H}), 6.26$ (br. s., 1H), 4.46 - $4.68(\mathrm{~m}, 2 \mathrm{H}), 3.73$ - $3.86(\mathrm{~m}, 4 \mathrm{H}), 3.70$ (br. s., $4 \mathrm{H})$

4-((4-Fluorobenzyl)amino)-6-morpholino-1,3,5-triazine-2-carbonitrile (Scheme 2, Int-3b) 
General procedure B2 from 4-chloro-N-(4-fluorobenzyl)-6-morpholino-1,3,5-triazin-2-amine (4, 12.35 mmol), potassium cyanide (0.885 g, $13.59 \mathrm{mmol})$, and DABCO (2.77 g, $24.71 \mathrm{mmol})$ gave 4-((4fluorobenzyl)amino)-6-morpholino-1,3,5-triazine-2-carbonitrile (2.7 g, 8.59 mmol, $69.5 \%$ yield).; ${ }^{1} \mathrm{H}$ NMR $\left(\mathrm{CDCl}_{3}, 500 \mathrm{MHz}\right) \delta 7.21$ - $7.30(\mathrm{~m}, 2 \mathrm{H}), 6.93$ - $7.06(\mathrm{~m}, 2 \mathrm{H}), 4.49$ - $4.61(\mathrm{~m}, 2 \mathrm{H}), 3.73$ - $3.86(\mathrm{~m}$, 4H), 3.69 (br. s., 4H)

\section{4-Chloro-N-isopentyl-6-morpholino-1,3,5-triazin-2-amine (Scheme 2, Int-2b)}

General procedure B1 from 2,4,6-trichloro-1,3,5-triazine (5, $27.1 \mathrm{mmol})$, 3-methylbutan-1-amine (3.15 ml, $27.1 \mathrm{mmol})$, DIPEA (9.48 ml, $54.2 \mathrm{mmol})$, and morpholine $(2.362 \mathrm{ml}, 27.1 \mathrm{mmol})$ gave 4-chloro-Nisopentyl-6-morpholino-1,3,5-triazin-2-amine (7.5 g, $26.2 \mathrm{mmol}, 97$ \% yield). Product could be recrystallized from hot EtOAc:Hexanes (2:1). ${ }^{1} \mathrm{H} \mathrm{NMR}\left(\mathrm{CDCl}_{3}, 500 \mathrm{MHz}\right) \delta 6.06$ (br. s., $\left.1 \mathrm{H}\right), 3.63$ - 3.88 $(\mathrm{m}, 8 \mathrm{H}), 3.33-3.46(\mathrm{~m}, 2 \mathrm{H}), 1.56-1.68(\mathrm{~m}, 1 \mathrm{H}), 1.44(\mathrm{q}, J=7.01 \mathrm{~Hz}, 2 \mathrm{H}), 0.91(\mathrm{~d}, J=6.36 \mathrm{~Hz}, 6 \mathrm{H})$

\section{4-(Isopentylamino)-6-morpholino-1,3,5-triazine-2-carbonitrile (Scheme 2, Int-3b)}

General procedure B2 from 4-chloro-N-isopentyl-6-morpholino-1,3,5-triazin-2-amine (3.24 g, 11.34 $\mathrm{mmol})$, potassium cyanide (0.812 $\mathrm{g}, 12.47 \mathrm{mmol})$, and DABCO (2.54 g, $22.68 \mathrm{mmol})$ gave 4(isopentylamino)-6-morpholino-1,3,5-triazine-2-carbonitrile (2.1 g, $7.60 \mathrm{mmol}, 68.7 \%$ yield).; ${ }^{1} \mathrm{H}$ NMR $\left(\mathrm{CDCl}_{3}, 500 \mathrm{MHz}\right) \delta 3.77$ - $3.91(\mathrm{~m}, 3 \mathrm{H}), 3.64$ - $3.77(\mathrm{~m}, 5 \mathrm{H}), 3.32$ - $3.49(\mathrm{~m}, 2 \mathrm{H}), 1.65(\mathrm{qd}, J=6.62$, $13.39 \mathrm{~Hz}, 1 \mathrm{H}), 1.39-1.53(\mathrm{~m}, 2 \mathrm{H}), 0.85-1.01(\mathrm{~m}, 6 \mathrm{H})$

\section{4-((2-Fluorobenzyl)(isopentyl)amino)-6-morpholino-1,3,5-triazine-2-carbonitrile (5)}

General Procedure B3 starting from 4-(isopentylamino)-6-morpholino-1,3,5-triazine-2-carbonitrile (100 mg, $0.362 \mathrm{mmol}$ ), sodium hydride (15.92 mg, $0.398 \mathrm{mmol}$ ), and 1-(bromomethyl)-2-fluorobenzene (68.4 mg, $0.362 \mathrm{mmol}$ ) gave 4-((2-fluorobenzyl) (isopentyl)amino)-6-morpholino-1,3,5-triazine-2-carbonitrile (67.6 mg, $0.176 \mathrm{mmol}, 48.6 \%$ yield) ${ }^{1} \mathrm{H} \mathrm{NMR}\left(\mathrm{CDCl}_{3}, 500 \mathrm{MHz}\right) \delta 7.24-7.30(\mathrm{~m}, 1 \mathrm{H}), 7.14-7.21$ (m, 1H), 7.01 - 7.12 (m, 2H), 4.86 (s, 1H), 4.80 (s, 1H), 3.82 (br. s., 1H), 3.78 (br. s., 2H), 3.70 - 3.75 (m, 
2H), 3.67 (br. s., 2H), 3.64 (br. s., 1H), 3.55 - 3.60 (m, 1H), 3.46 - 3.52 (m, 1H), 1.56 (qd, J = 6.60, 16.38 $\mathrm{Hz}, 1 \mathrm{H}), 1.41-1.49(\mathrm{~m}, 2 \mathrm{H}), 0.91(\mathrm{t}, \mathrm{J}=7.34 \mathrm{~Hz}, 6 \mathrm{H}) ;{ }^{13} \mathrm{C} \mathrm{NMR}\left(\mathrm{CDCl}_{3}, 126 \mathrm{MHz}\right) \delta 164.2,164.1$, $163.6,163.4,161.9,161.8,160.0,159.8,130.0,129.9,129.2,129.0,128.9,124.3,124.2,115.6,115.5$, $115.4,115.2,66.7,66.4,45.6,45.0,43.9,43.4,43.3,36.26,35.43,26.01,25.88,22.51,22.47$; HRMSESI $(\mathrm{m} / \mathrm{z}):[\mathrm{M}+\mathrm{H}]^{+}$calcd for $\mathrm{C}_{20} \mathrm{H}_{25} \mathrm{FN}_{6} \mathrm{O}, 385.2147$; found, 385.2150

\section{4-((3-Fluorobenzyl)(isopentyl)amino)-6-morpholino-1,3,5-triazine-2-carbonitrile (6)}

General Procedure B3 starting from 4-(isopentylamino)-6-morpholino-1,3,5-triazine-2-carbonitrile (100 mg, $0.362 \mathrm{mmol})$, sodium hydride (21.71 mg, $0.543 \mathrm{mmol})$, and 1-(bromomethyl)-3-fluorobenzene (68.4 mg, $0.362 \mathrm{mmol}$ ) gave 4-((3-fluorobenzyl)(isopentyl)amino)-6-morpholino-1,3,5-triazine-2-carbonitrile (104 mg, $0.271 \mathrm{mmol}, 74.8 \%$ yield); ${ }^{1} \mathrm{H}$ NMR $\left(\mathrm{CDCl}_{3}, 500 \mathrm{MHz}\right) \delta 7.21$ - $7.32(\mathrm{~m}, 1 \mathrm{H}), 6.97$ - 7.02 (m, 1H), 6.93 - $6.97(\mathrm{~m}, 1 \mathrm{H}), 6.91(\mathrm{~d}, \mathrm{~J}=9.29 \mathrm{~Hz}, 1 \mathrm{H}), 4.79(\mathrm{~s}, 1 \mathrm{H}), 4.72(\mathrm{~s}, 1 \mathrm{H}), 3.81-3.85(\mathrm{~m}, 1 \mathrm{H}), 3.79$ (br. s., 2H), 3.71 - 3.75 (m, 2H), 3.68 (br. s., 1H), 3.60 - $3.66(\mathrm{~m}, 2 \mathrm{H}), 3.51$ - $3.60(\mathrm{~m}, 1 \mathrm{H}), 3.41$ - 3.50 (m, 1H), $1.48-1.60(\mathrm{~m}, 1 \mathrm{H}), 1.39-1.48(\mathrm{~m}, 2 \mathrm{H}), 0.90(\mathrm{t}, \mathrm{J}=7.09 \mathrm{~Hz}, 6 \mathrm{H}) ;{ }^{13} \mathrm{C} \mathrm{NMR}\left(\mathrm{CDCl}_{3}, 126 \mathrm{MHz}\right) \delta$ $164.2,164.0,163.9,163.6,163.4,161.9,152.0,151.9,140.2,140.1,140.0,139.9,130.1,130.0,123.2$, $122.8,122.7,115.5,114.4,114.3,114.2,114.1,114.0,66.6,66.3,49.4,49.3,45.5,44.8,43.9,43.8,43.4$, 36.2, 35.4, 26.0, 25.8, 22.5, 22.4; HRMS-ESI (m/z): $[\mathrm{M}+\mathrm{H}]^{+}$calcd for $\mathrm{C}_{20} \mathrm{H}_{25} \mathrm{FN}_{6} \mathrm{O}, 385.2147$; found, 385.2151

\section{4-((2,6-Difluorobenzyl)(isopentyl)amino)-6-morpholino-1,3,5-triazine-2-carbonitrile (7)}

General Procedure B3 starting from 4-(isopentylamino)-6-morpholino-1,3,5-triazine-2-carbonitrile (100 mg, $0.362 \mathrm{mmol})$, sodium hydride (28.9 mg, $0.724 \mathrm{mmol})$, and 2-(bromomethyl)-1,3-difluorobenzene (74.9 mg, $0.362 \mathrm{mmol})$ gave 4-((2,6-difluorobenzyl)(isopentyl)amino)-6-morpholino-1,3,5-triazine-2carbonitrile (51.6 mg, $0.128 \mathrm{mmol}, 35.4 \%$ yield); ${ }^{1} \mathrm{H}$ NMR $\left(\mathrm{CDCl}_{3}, 500 \mathrm{MHz}\right) \delta 7.20-7.34(\mathrm{~m}, 1 \mathrm{H})$, 6.79 - $6.94(\mathrm{~m}, 2 \mathrm{H}), 4.95(\mathrm{~s}, 1 \mathrm{H}), 4.85(\mathrm{~s}, 1 \mathrm{H}), 3.74-3.86(\mathrm{~m}, 4 \mathrm{H}), 3.66-3.74(\mathrm{~m}, 4 \mathrm{H}), 3.55(\mathrm{t}, \mathrm{J}=7.80$ $\mathrm{Hz}, 1 \mathrm{H}), 3.43$ (t, J = 7.80 Hz, 1H), $1.46-1.59(\mathrm{~m}, 1 \mathrm{H}), 1.34-1.46(\mathrm{~m}, 2 \mathrm{H}), 0.90(\mathrm{~d}, \mathrm{~J}=6.36 \mathrm{~Hz}, 3 \mathrm{H})$, 
$0.87(\mathrm{~d}, \mathrm{~J}=6.85 \mathrm{~Hz}, 3 \mathrm{H}) ;{ }^{13} \mathrm{C} \mathrm{NMR}\left(\mathrm{CDCl}_{3}, 126 \mathrm{MHz}\right) \delta 163.8,163.7,163.6,163.4,163.0,163.0,162.9$, $162.8,161.0,160.9,160.8,151.9,151.6,129.8,129.7,129.6,129.5,115.6,112.9,111.5,111.4,111.3$, 66.7, 66.4, 45.1, 44.2, 43.9, 43.4, 37.9, 36.1, 35.2, 26.0, 25.9, 22.5; HRMS-ESI (m/z): $[\mathrm{M}+\mathrm{H}]^{+}$calcd for $\mathrm{C}_{20} \mathrm{H}_{24} \mathrm{~F}_{2} \mathrm{~N}_{6} \mathrm{O}$, 403.2058; found, 403.2055

\section{4-((3,4-Difluorobenzyl)(isopentyl)amino)-6-morpholino-1,3,5-triazine-2-carbonitrile (8)}

General Procedure B3 starting from 4-(isopentylamino)-6-morpholino-1,3,5-triazine-2-carbonitrile (50 $\mathrm{mg}, 0.181 \mathrm{mmol})$, sodium hydride $(7.96 \mathrm{mg}, 0.199 \mathrm{mmol})$, and 4-(bromomethyl)-1,2-difluorobenzene (0.023 ml, $0.181 \mathrm{mmol})$ gave 4-((3,4-difluorobenzyl)(isopentyl)amino)-6-morpholino-1,3,5-triazine-2carbonitrile (62 mg, $0.154 \mathrm{mmol}, 85 \%$ yield); ${ }^{1} \mathrm{H} \mathrm{NMR}\left(\mathrm{CDCl}_{3}, 500 \mathrm{MHz}\right) \delta 7.10(\mathrm{q}, \mathrm{J}=8.80 \mathrm{~Hz}, 1 \mathrm{H})$, $7.00-7.06(\mathrm{~m}, 1 \mathrm{H}), 6.89-6.98(\mathrm{~m}, 1 \mathrm{H}), 4.73(\mathrm{~s}, 1 \mathrm{H}), 4.68(\mathrm{~s}, 1 \mathrm{H}), 3.76$ - $3.87(\mathrm{~m}, 3 \mathrm{H}), 3.62$ - $3.74(\mathrm{~m}$, 5H), 3.50 - $3.56(\mathrm{~m}, 1 \mathrm{H}), 3.41-3.48(\mathrm{~m}, 1 \mathrm{H}), 1.48-1.58(\mathrm{~m}, 1 \mathrm{H}), 1.38-1.46(\mathrm{~m}, 2 \mathrm{H}), 0.90(\mathrm{t}, \mathrm{J}=6.60$ $\mathrm{Hz}, 6 \mathrm{H}) ;{ }^{13} \mathrm{C} \mathrm{NMR}\left(\mathrm{CDCl}_{3}, 126 \mathrm{MHz}\right) \delta 164.2,164.0,163.6,163.5,152.1,151.9,151.3,150.7,150.6$, $149.3,148.8,148.6,148.5,134.5,134.4,123.7,123.6,123.2,123.1,117.4,117.3,117.2,116.6,116.4$ $116.2,116.1,115.5,115.4,66.6,66.3,48.9,45.4,44.8,44.0,43.9,43.4,36.2,35.4,31.5,26.0,25.9,22.6$, 22.5, 22.4; ${ }^{19} \mathrm{~F} \mathrm{NMR}\left(\mathrm{CDCl}_{3}, 470 \mathrm{MHz}\right) \delta$-137.2 (m), -139.53 (m); HRMS-ESI (m/z): $[\mathrm{M}+\mathrm{H}]^{+}$calcd for, $\mathrm{C}_{20} \mathrm{H}_{24} \mathrm{~F}_{2} \mathrm{~N}_{6} \mathrm{O}$; 403.2058; found, 403.2055

\section{4-((2,4-Difluorobenzyl)(isopentyl)amino)-6-morpholino-1,3,5-triazine-2-carbonitrile (9)}

General Procedure B3 starting from 4-(isopentylamino)-6-morpholino-1,3,5-triazine-2-carbonitrile (75 mg, $0.271 \mathrm{mmol}$ ), sodium hydride (11.94 mg, $0.299 \mathrm{mmol})$, and 1-(bromomethyl)-2,4-difluorobenzene (0.033 ml, $0.271 \mathrm{mmol})$ gave 4-((2,4-difluorobenzyl)(isopentyl)amino)-6-morpholino-1,3,5-triazine-2carbonitrile (86 mg, $0.214 \mathrm{mmol}, 79 \%$ yield); ${ }^{1} \mathrm{H} \mathrm{NMR}\left(\mathrm{CDCl}_{3}, 500 \mathrm{MHz}\right) \delta 7.13-7.33(\mathrm{~m}, 1 \mathrm{H}), 6.78$ $6.86(\mathrm{~m}, 2 \mathrm{H}), 4.80(\mathrm{~s}, 1 \mathrm{H}), 4.75(\mathrm{~s}, 1 \mathrm{H}), 3.76-3.86(\mathrm{~m}, 3 \mathrm{H}), 3.63$ - $3.74(\mathrm{~m}, 5 \mathrm{H}), 3.52$ - $3.60(\mathrm{~m}, 1 \mathrm{H})$ 3.45 - $3.52(\mathrm{~m}, 1 \mathrm{H}), 1.51-1.60(\mathrm{~m}, 1 \mathrm{H}), 1.40-1.49(\mathrm{~m}, 2 \mathrm{H}), 0.92(\mathrm{t}, \mathrm{J}=6.36 \mathrm{~Hz}, 6 \mathrm{H}) ;{ }^{13} \mathrm{C} \mathrm{NMR}\left(\mathrm{CDCl}_{3}\right.$, $126 \mathrm{MHz}) \delta 164.2,164.1,163.6,163.5,163.4,163.3,163.1,162.0,161.9,161.8,161.7,161.4,161.3$, 
$161.2,160.0,159.9,159.8,159.7,152.0,151.8,131.2,131.1,130.0,129.9,120.4,120.3,120.2,115.5$, 111.6, 111.5, 111.4, 111.3, 104.0, 103.9, 103.8, 103.7, 103.6, 103.5, 66.7, 66.4, 45.6, 45.1, 44.0, 43.4, 42.9, 42.8, 36.3, 35.5, 26.0, 25.9, 22.5, 22.4; ${ }^{19} \mathrm{~F} \mathrm{NMR}\left(\mathrm{CDCl}_{3}, 470 \mathrm{MHz}\right) \delta-114.58(\mathrm{~m}),-111.04(\mathrm{~m})$; HRMS-ESI (m/z): [M+H] $]^{+}$calcd for, $\mathrm{C}_{20} \mathrm{H}_{24} \mathrm{~F}_{2} \mathrm{~N}_{6} \mathrm{O}$; 403.2058; found, 403.2054

\section{4-(Benzyl(isopentyl)amino)-6-morpholino-1,3,5-triazine-2-carbonitrile (10)}

General Procedure B3 starting from 4-(isopentylamino)-6-morpholino-1,3,5-triazine-2-carbonitrile (100 $\mathrm{mg}, 0.362 \mathrm{mmol}$ ) and (bromomethyl)benzene (92.8 mg, $0.543 \mathrm{mmol}$ ) gave 4-(benzyl(isopentyl)amino)-6morpholino-1,3,5-triazine-2-carbonitrile (89.1 mg, $0.243 \mathrm{mmol}, 67.2 \%$ yield); ${ }^{1} \mathrm{H} \mathrm{NMR}\left(\mathrm{CDCl}_{3}, 500\right.$ MHz) $\delta 7.37-7.16(\mathrm{~m}, 4 \mathrm{H}), 4.78(\mathrm{~d}, \mathrm{~J}=27.6 \mathrm{~Hz}, 2 \mathrm{H}), 3.89-3.58(\mathrm{~m}, 8 \mathrm{H}), 3.58-3.50(\mathrm{~m}, 1 \mathrm{H}), 3.50-$ $3.39(\mathrm{~m}, 1 \mathrm{H}), 1.54(\mathrm{~m}, 1 \mathrm{H}), 1.44(\mathrm{qd}, \mathrm{J}=7.0,1.4 \mathrm{~Hz}, 2 \mathrm{H}), 0.90(\mathrm{dd}, \mathrm{J}=7.8,6.5 \mathrm{~Hz}, 6 \mathrm{H}).) ;{ }^{13} \mathrm{C} \mathrm{NMR}$ $\left(\mathrm{CDCl}_{3}, 126 \mathrm{MHz}\right) \delta 164.05,163.69,163.54,152.05,137.43,137.31,128.61,128.58,127.77,127.50$, $127.39,127.32,115.61,49.74,45.29,44.67,36.23,35.37,26.05,25.91,22.56,22.51$. HRMS-ESI (m/z): $[\mathrm{M}+\mathrm{H}]^{+}$calcd for $\mathrm{C}_{20} \mathrm{H}_{26} \mathrm{~N}_{6} \mathrm{O}, 367.2241$; found, 367.2284

\section{4-(Isopentyl(phenethyl)amino)-6-morpholino-1,3,5-triazine-2-carbonitrile (11)}

General Procedure B3 starting from 4-(isopentylamino)-6-morpholino-1,3,5-triazine-2-carbonitrile (100 $\mathrm{mg}, 0.362 \mathrm{mmol})$, sodium hydride $(28.9 \mathrm{mg}, 0.724 \mathrm{mmol})$, and (2-bromoethyl)benzene (49.2 $\mu 1,0.362$ mmol) gave 4-(isopentyl(phenethyl)amino)-6-morpholino-1,3,5-triazine-2-carbonitrile (15.2 mg, 0.040 mmol, $11.04 \%$ yield); ${ }^{1} \mathrm{H} \mathrm{NMR}\left(\mathrm{CDCl}_{3}, 500 \mathrm{MHz}\right) \delta 7.27(\mathrm{dd}, J=4.65,6.11 \mathrm{~Hz}, 1 \mathrm{H}), 7.21(\mathrm{~d}, J=6.85$ Hz, 1H), 4.75 (br. s., 7H), 3.78 (br. s., 3H), 3.58 - 3.73 (m, 4H), 3.42 - 3.52 (m, 1H), 3.33 - 3.42 (m, 1H), 2.85 (br. s., 1H), 2.03 (br. s., 2H), 1.49 - 1.62 (m, 1H), $1.36-1.47(\mathrm{~m}, 2 \mathrm{H}), 0.77-1.00(\mathrm{~m}, 6 \mathrm{H}) ;{ }^{13} \mathrm{C}$ NMR $\left(\mathrm{CDCl}_{3}, 126 \mathrm{MHz}\right) \delta 163.5,163.4,151.7,138.9,138.8,128.8,128.6,128.5,128.4,126.4,126.3,115.6$, $66.6,66.3,49.2,48.8,46.0,45.9,43.8,43.3,39.0,38.0,36.6,35.9,34.3,33.5,25.9,25.8,22.5,22.4$; HRMS-ESI (m/z): $[\mathrm{M}+\mathrm{H}]^{+}$calcd for, $\mathrm{C}_{21} \mathrm{H}_{28} \mathrm{~N}_{6} \mathrm{O}$; 381.2403; found, 381.2401 


\section{4-(Isopentyl(3-phenylpropyl)amino)-6-morpholino-1,3,5-triazine-2-carbonitrile (12)}

General Procedure B3 starting from4-(isopentylamino)-6-morpholino-1,3,5-triazine-2-carbonitrile (100 $\mathrm{mg}, 0.362 \mathrm{mmol})$, sodium hydride $(21.71 \mathrm{mg}, 0.543 \mathrm{mmol})$, and (3-bromopropyl)benzene (72.0 $\mathrm{mg}, 0.362$ mmol) gave 4-(isopentyl(3-phenylpropyl)amino)-6-morpholino-1,3,5-triazine-2-carbonitrile (113 mg, $0.286 \mathrm{mmol}, 79 \%$ yield); ${ }^{1} \mathrm{H}$ NMR $\left(\mathrm{CDCl}_{3}, 500 \mathrm{MHz}\right) \delta 7.25-7.34(\mathrm{~m}, 2 \mathrm{H}), 7.14-7.25(\mathrm{~m}, 3 \mathrm{H}), 3.80$ (br. s., 1H), 3.76 (br. s., 2H), 3.69 - 3.73 (m, 2H), 3.67 (br. s., 1H), 3.63 (br. s., 1H), 3.50 - 3.58 (m, 2H), $3.41-3.50(\mathrm{~m}, 3 \mathrm{H}), 2.60-2.70(\mathrm{~m}, 2 \mathrm{H}), 1.87-1.97(\mathrm{~m}, 2 \mathrm{H}), 1.49-1.62(\mathrm{~m}, 1 \mathrm{H}), 1.35-1.48(\mathrm{~m}, 2 \mathrm{H})$, $0.93(\mathrm{t}, J=7.58 \mathrm{~Hz}, 6 \mathrm{H}) ;{ }^{13} \mathrm{C} \mathrm{NMR}\left(\mathrm{CDCl}_{3}, 126 \mathrm{MHz}\right) \delta 163.4,163.3,151.7,141.3,128.3,128.2,125.9$, $125.8,115.6,66.6,66.3,46.8,46.4,45.7,45.5,43.8,43.7,43.3,43.1,36.6,35.8,33.2,32.9,29.2,28.4$, 26.0, 25.8, 22.5; HRMS-ESI (m/z): [M+H] $]^{+}$calcd for, $\mathrm{C}_{22} \mathrm{H}_{30} \mathrm{~N}_{6} \mathrm{O} ; 395.2559$; found, 395.2557

\section{4-(Isopentyl(quinolin-8-ylmethyl)amino)-6-morpholino-1,3,5-triazine-2-carbonitrile (13)}

General Procedure B3 starting from 4-(isopentylamino)-6-morpholino-1,3,5-triazine-2-carbonitrile (100 mg, $0.362 \mathrm{mmol})$, sodium hydride (28.9 mg, $0.724 \mathrm{mmol})$, and 8-(bromomethyl)quinoline (80 mg, 0.362 mmol) gave 4-(isopentyl(quinolin-8-ylmethyl)amino)-6-morpholino-1,3,5-triazine-2-carbonitrile (41.3 mg, 0.099 mmol, $27.3 \%$ yield); ${ }^{1} \mathrm{H} \mathrm{NMR}\left(\mathrm{CDCl}_{3}, 500 \mathrm{MHz}\right) \delta 8.90$ - 8.98 (m, 1H), 8.14 - $8.22(\mathrm{~m}, 1 \mathrm{H})$, $7.74(\mathrm{~d}, J=7.83 \mathrm{~Hz}, 1 \mathrm{H}), 7.40-7.52(\mathrm{~m}, 3 \mathrm{H}), 5.52$ (s, 1H), 5.48 (s, 1H), 3.82 (br. s., 2H), 3.73 (br. s., 3H), 3.66 - 3.70 (m, 1H), 3.57 - 3.66 (m, 2H), 3.45 (br. s., 2H), 1.49 - 1.63 (m, 3H), 0.90 (d, J= 5.87 Hz, 3H), $0.88(\mathrm{~d}, J=5.87 \mathrm{~Hz}, 3 \mathrm{H}) ;{ }^{13} \mathrm{C} \mathrm{NMR}\left(\mathrm{CDCl}_{3}, 126 \mathrm{MHz}\right) \delta 164.3,163.6,163.5,151.9,149.4,146.5$, $146.3,136.4,136.2,135.4,135.1,128.2,127.2,127.1,126.9,126.4,126.3,121.1,115.7,115.6,66.7$, 66.6, 66.4, 66.2, 46.1 , 46.0, 45.6, 43.9, 43.8, 43.4, 43.2, 36.5, 35.6, 26.1, 25.9, 22.5; HRMS-ESI (m/z): $[\mathrm{M}+\mathrm{H}]^{+}$calcd for, $\mathrm{C}_{23} \mathrm{H}_{27} \mathrm{~N}_{7} \mathrm{O} ; 4$ 418.2355; found, 418.2365

\section{4-(Isopentyl(naphthalen-1-ylmethyl)amino)-6-morpholino-1,3,5-triazine-2-carbonitrile (14)}

General Procedure B3 starting from 4-(isopentylamino)-6-morpholino-1,3,5-triazine-2-carbonitrile (100 $\mathrm{mg}, 0.362 \mathrm{mmol})$, sodium hydride $(21.71 \mathrm{mg}, 0.543 \mathrm{mmol})$, and 1-(bromomethyl)naphthalene (80 $\mathrm{mg}$, 
$0.362 \mathrm{mmol}$ ) gave 4-(isopentyl(naphthalen-1-ylmethyl)amino)-6-morpholino-1,3,5-triazine-2-carbonitrile (41.3 mg, $0.099 \mathrm{mmol}, 27.4 \%$ yield); ${ }^{1} \mathrm{H}$ NMR $\left(\mathrm{CDCl}_{3}, 500 \mathrm{MHz}\right) \delta 7.96-8.05(\mathrm{~m}, 1 \mathrm{H}), 7.85$ - 7.93 (m, 1H), $7.81(\mathrm{~d}, J=8.31 \mathrm{~Hz}, 1 \mathrm{H}), 7.48-7.57(\mathrm{~m}, 2 \mathrm{H}), 7.39-7.45(\mathrm{~m}, 1 \mathrm{H}), 7.22$ - $7.29(\mathrm{~m}, 1 \mathrm{H}), 5.29(\mathrm{~s}, 1 \mathrm{H})$, 5.22 (s, 1H), 3.85 (br. s., 1H), 3.79 (br. s., 2H), 3.73 (br. s., 2H), 3.68 (br. s., 1H), 3.59 (br. s., 1H), 3.50 $3.57(\mathrm{~m}, 2 \mathrm{H}), 3.40-3.48(\mathrm{~m}, 1 \mathrm{H}), 1.45-1.56(\mathrm{~m}, 1 \mathrm{H}), 1.36-1.45(\mathrm{~m}, 2 \mathrm{H}), 0.86(\mathrm{~d}, J=6.85 \mathrm{~Hz}, 3 \mathrm{H})$, $0.84(\mathrm{~d}, J=6.36 \mathrm{~Hz}, 3 \mathrm{H}) ;{ }^{13} \mathrm{C} \mathrm{NMR}\left(\mathrm{CDCl}_{3}, 126 \mathrm{MHz}\right) \delta 194.0,193.9,164.2,164.0,163.6,163.5,152.6$, 152.6, 152.0, 151.9, 143.5, 133.4, 133.1, 133.0, 132.9, 126.3, 125.8, 124.7, 124.1, 123.9, 115.6, 115.5, 82.3, 66.7, 66.4, 49.0, 45.5, 44.7, 44.3, 44.2, 44.0, 43.9, 43.4, 38.9, 28.3, 28.2, 22.5; HRMS-ESI (m/z): $[\mathrm{M}+\mathrm{H}]^{+}$calcd for, $\mathrm{C}_{24} \mathrm{H}_{28} \mathrm{~N}_{6} \mathrm{O} ; 417.2397$; found, 417.2398

\section{4-((1-(4-Fluorophenyl)ethyl)(isopentyl)amino)-6-morpholino-1,3,5-triazine-2-carbonitrile (49)}

General Procedure A4 from 4-chloro-N-(1-(4-fluorophenyl)ethyl)-N-isopentyl-6-morpholino-1,3,5triazin-2-amine $(200 \mathrm{mg}, 0.490 \mathrm{mmol}), \mathrm{DABCO}(110 \mathrm{mg}, 0.981 \mathrm{mmol})$, and potassium cyanide $(35.1 \mathrm{mg}$, $0.539 \mathrm{mmol})$ in DMSO (2 ml) gave 4-((1-(4-fluorophenyl)ethyl)(isopentyl)amino)-6-morpholino-1,3,5triazine-2-carbonitrile (72 mg, $0.181 \mathrm{mmol}, 36.9 \%$ yield); ${ }^{1} \mathrm{H}$ NMR $\left(\mathrm{CDCl}_{3}, 500 \mathrm{MHz}\right) \delta 7.21-7.30$ (m, 2H), $7.01(\mathrm{t}, J=8.56 \mathrm{~Hz}, 2 \mathrm{H}), 6.15(\mathrm{q}, J=6.85 \mathrm{~Hz}, 0.5 \mathrm{H}), 5.93(\mathrm{q}, J=6.85 \mathrm{~Hz}, 0.5 \mathrm{H}), 3.67$ - $3.85(\mathrm{~m}$, 8H), $3.31-3.41(\mathrm{~m}, 0.5 \mathrm{H}), 3.21-3.29(\mathrm{~m}, 0.5 \mathrm{H}), 3.02-3.18(\mathrm{~m}, 1 \mathrm{H}), 1.51-1.63(\mathrm{~m}, 3 \mathrm{H}), 1.38$ - $1.49(\mathrm{~m}$, 1H), 1.29 - $1.38(\mathrm{~m}, 1 \mathrm{H}), 1.11-1.23(\mathrm{~m}, 1 \mathrm{H}), 0.76-0.85(\mathrm{~m}, 6 \mathrm{H}) ;{ }^{13} \mathrm{C} \mathrm{NMR}\left(\mathrm{CDCl}_{3}, 126 \mathrm{MHz}\right) \delta 163.7$, $163.6,163.5,163.4,163.0,161.1,152.0,151.7,136.6,136.4,129.0,128.7,128.6,115.6,115.3,115.1$, $66.7,66.4,52.2,51.9,44.0,43.4,42.5,41.9,37.7,36.6,26.7,26.4,22.5,22.4,22.3,17.0,16.9$; HRMSESI (m/z): $[\mathrm{M}+\mathrm{H}]^{+}$calcd for, $\mathrm{C}_{21} \mathrm{H}_{27} \mathrm{FN}_{6} \mathrm{O}$; 399.2303; found, 399.2302

\section{N-(1-(4-Fluorophenyl)ethyl)-3-methylbutan-1-amine (Scheme 1, Int-2b)}

1-(4-Fluorophenyl)ethan-1-one (0.5 ml, $4.12 \mathrm{mmol})$, 3-methylbutan-1-amine (1.434 ml, $12.36 \mathrm{mmol})$, and titanium(iv) isopropoxide $(1.810 \mathrm{ml}, 6.18 \mathrm{mmol})$ were added to a flask containing $\mathrm{MeOH}(5 \mathrm{ml})$ and allowed to stir at room temperature overnight. The next morning, the reaction was cooled to $0^{\circ} \mathrm{C}$ and 
sodium borohydride (467 $\mathrm{mg}, 12.36 \mathrm{mmol}$ ) was added slowly. The reaction was allowed to warm back to room temperature and stirred for 4-6 h. Reaction was then quenched slowly with $\mathrm{H}_{2} \mathrm{O}$ and solvent was removed in vacuo. Crude residue was taken up in aqueous bicarbonate, extracted with EtOAc 3x, dried over $\mathrm{MgSO}_{4}$ and concentrated to afford N-(1-(4-fluorophenyl)ethyl)-3-methylbutan-1-amine (745 mg, $3.56 \mathrm{mmol}, 86 \%$ yield $) ;{ }^{1} \mathrm{H} \mathrm{NMR}\left(\mathrm{CDCl}_{3}, 500 \mathrm{MHz}\right) \delta 7.20-7.30(\mathrm{~m}, 2 \mathrm{H}), 7.13(\mathrm{~d}, J=4.40 \mathrm{~Hz}, 1 \mathrm{H})$, 7.00 (br. t, $J=7.80 \mathrm{~Hz}, 2 \mathrm{H}), 6.14$ (br. d, $J=6.80 \mathrm{~Hz}, 1 \mathrm{H}), 4.92-5.08(\mathrm{~m}, 1 \mathrm{H}), 4.76-4.92(\mathrm{~m}, 2 \mathrm{H}), 4.58$ $4.73(\mathrm{~m}, 2 \mathrm{H}), 3.19$ - $3.39(\mathrm{~m}, 1 \mathrm{H}), 2.98$ - $3.17(\mathrm{~m}, 1 \mathrm{H}), 1.52$ - $1.59(\mathrm{~m}, 3 \mathrm{H}), 1.39-1.48(\mathrm{~m}, 1 \mathrm{H}), 1.28$ $1.37(\mathrm{~m}, 1 \mathrm{H}), 1.12-1.20(\mathrm{~m}, 1 \mathrm{H}), 0.75-0.87(\mathrm{~m}, 6 \mathrm{H})$

\section{6-Chloro-N2-(1-(4-fluorophenyl)ethyl)-N2-isopentyl-N4-(oxetan-3-yl)-1,3,5-triazine-2,4-diamine}

\section{(Scheme 2, Int-2b)}

General procedure B1 from 2,4,6-trichloro-1,3,5-triazine (100 mg, $0.542 \mathrm{mmol}), \mathrm{N}-(1-(4-$ fluorophenyl)ethyl)-3-methylbutan-1-amine (114 mg, $0.542 \mathrm{mmol})$, DIPEA (189 $\mu 1,1.085 \mathrm{mmol})$, and oxetan-3-amine (39.6 mg, $0.542 \mathrm{mmol}$ ) gave 6-chloro-N2-(1-(4-fluorophenyl)ethyl)-N2-isopentyl-N4(oxetan-3-yl)-1,3,5-triazine-2,4-diamine (155 mg, $0.394 \mathrm{mmol}, 72.6 \%$ yield); ${ }^{1} \mathrm{H} \mathrm{NMR}\left(\mathrm{CDCl}_{3}, 500\right.$ MHz) $\delta 7.20-7.30(\mathrm{~m}, 2 \mathrm{H}), 7.13(\mathrm{~d}, \mathrm{~J}=4.40 \mathrm{~Hz}, 1 \mathrm{H}), 7.00$ (br. t, $\mathrm{J}=7.80 \mathrm{~Hz}, 2 \mathrm{H}), 6.14$ (br. d, J = 6.80 $\mathrm{Hz}, 1 \mathrm{H}), 4.92$ - $5.08(\mathrm{~m}, 1 \mathrm{H}), 4.76$ - $4.92(\mathrm{~m}, 2 \mathrm{H}), 4.58$ - $4.73(\mathrm{~m}, 2 \mathrm{H}), 3.19$ - $3.39(\mathrm{~m}, 1 \mathrm{H}), 2.98$ - 3.17 (m, 1H), $1.52-1.59(\mathrm{~m}, 3 \mathrm{H}), 1.39-1.48(\mathrm{~m}, 1 \mathrm{H}), 1.28-1.37(\mathrm{~m}, 1 \mathrm{H}), 1.12-1.20(\mathrm{~m}, 1 \mathrm{H}), 0.75-0.87$ $(\mathrm{m}, 6 \mathrm{H})$

\section{4-((1-(4-Fluorophenyl)ethyl)(isopentyl)amino)-6-(oxetan-3-ylamino)-1,3,5-triazine-2-carbonitrile} (50)

General procedure B2 from 6-chloro-N2-(1-(4-fluorophenyl)ethyl)-N2-isopentyl-N4-(oxetan-3-yl)-1,3,5triazine-2,4-diamine (155 mg, $0.394 \mathrm{mmol})$, potassium cyanide $(28.2 \mathrm{mg}, 0.433 \mathrm{mmol})$, and DABCO (88 mg, $0.787 \mathrm{mmol}$ ) gave 4-((1-(4-fluorophenyl)ethyl)(isopentyl)amino)-6-(oxetan-3-ylamino)-1,3,5triazine-2-carbonitrile (72 mg, $0.187 \mathrm{mmol}, 47.6 \%$ yield); ${ }^{1} \mathrm{H}$ NMR (DMSO-d 6 , $\left.400 \mathrm{MHz}\right) \delta 7.30-7.37$ 
(m, 2H), 7.12 (t, $J=8.80 \mathrm{~Hz}, 2 \mathrm{H}), 5.91$ (br. s., $1 \mathrm{H}), 4.86$ (br. s., $1 \mathrm{H}), 4.70$ (br. s., $2 \mathrm{H}), 4.53$ (br. s., $2 \mathrm{H}$ ), 3.31 (br. s., 1H), 3.20 (br. s., 1H), 2.99 (br. s., 1H), 1.55 (d, J= 7.04 Hz, 3H), 1.40 - 1.50 (m, 1H), 1.28 $1.40(\mathrm{~m}, 1 \mathrm{H}), 1.05-1.15(\mathrm{~m}, 1 \mathrm{H}), 0.81(\mathrm{~d}, J=7.04 \mathrm{~Hz}, 3 \mathrm{H}), 0.79(\mathrm{~d}, J=7.00 \mathrm{~Hz}, 3 \mathrm{H}) ;{ }^{13} \mathrm{C} \mathrm{NMR}\left(\mathrm{CDCl}_{3}\right.$, $126 \mathrm{MHz}) \delta 178.1,163.8,163.6,163.4,163.2,162.8,160.8,151.4,135.9,128.8,128.5,128.4,115.2$, $115.1,114.9,78.1,77.6,72.2,61.3,52.1,51.8,45.6,42.2,41.8,40.1,39.9,39.6,39.4,37.3,36.5,26.4$, 26.1, 22.2, 16.8; $[\mathrm{M}+\mathrm{H}]^{+}$calcd for, $\mathrm{C}_{20} \mathrm{H}_{25} \mathrm{FN}_{6} \mathrm{O} ; 385.2147$; found, 385.2149

\section{4-(Isopentyl(pyridin-2-ylmethyl)amino)-6-morpholino-1,3,5-triazine-2-carbonitrile (15)}

General procedure B3 from 4-(isopentylamino)-6-morpholino-1,3,5-triazine-2-carbonitrile (50 mg, 0.181 mmol), sodium hydride (14.47 mg, $0.362 \mathrm{mmol}$ ), and 2-(bromomethyl)pyridine, $\mathrm{HBr}$ (45.8 mg, 0.181 mmol) gave 4-(isopentyl(pyridin-2-ylmethyl)amino)-6-morpholino-1,3,5-triazine-2-carbonitrile (16.8 mg,

$0.046 \mathrm{mmol}, 25.3 \%$ yield); ${ }^{1} \mathrm{H} \mathrm{NMR}\left(\mathrm{CDCl}_{3}, 500 \mathrm{MHz}\right) \delta 8.55(\mathrm{~d}, J=4.40 \mathrm{~Hz}, 1 \mathrm{H}), 7.64(\mathrm{~d}, J=6.85 \mathrm{~Hz}$, 1H), $7.23(\mathrm{~d}, J=7.83 \mathrm{~Hz}, 0.5 \mathrm{H}), 7.16-7.22(\mathrm{~m}, 1 \mathrm{H}), 7.11(\mathrm{~d}, J=7.83 \mathrm{~Hz}, 0.5 \mathrm{H}), 4.90(\mathrm{~s}, 1 \mathrm{H}), 4.84(\mathrm{~s}$, $1 \mathrm{H}), 3.75$ - $3.85(\mathrm{~m}, 3 \mathrm{H}), 3.70$ - $3.75(\mathrm{~m}, 2 \mathrm{H}), 3.63$ - $3.70(\mathrm{~m}, 2 \mathrm{H}), 3.54$ - $3.61(\mathrm{~m}, 3 \mathrm{H}), 1.51$ - $1.64(\mathrm{~m}$, 1H), $1.42-1.51(\mathrm{~m}, 2 \mathrm{H}), 0.93(\mathrm{~d}, J=6.36 \mathrm{~Hz}, 3 \mathrm{H}), 0.90(\mathrm{~d}, J=6.85 \mathrm{~Hz}, 3 \mathrm{H}) ;{ }^{13} \mathrm{C} \mathrm{NMR}\left(\mathrm{CDCl}_{3}, 126\right.$ MHz) $\delta 164.2,164.0,163.6,163.4,157.6,157.4,152.0,151.9,149.3,136.7,136.6,122.4,122.2,122.0$, $120.8,115.6,115.5,66.7,66.6,66.4,66.3,52.3,51.9,46.3,45.6,44.0,43.4,36.4,35.5,26.0,25.9,22.6$, 22.5; HRMS-ESI (m/z): $[\mathrm{M}+\mathrm{H}]^{+}$calcd for, $\mathrm{C}_{19} \mathrm{H}_{25} \mathrm{~N}_{7} \mathrm{O}$; 368.2193; found, 368.2200

\section{4-(Isopentyl(pyridin-3-ylmethyl)amino)-6-morpholino-1,3,5-triazine-2-carbonitrile (16)}

General procedure B3 from 4-(isopentylamino)-6-morpholino-1,3,5-triazine-2-carbonitrile (50 mg, 0.181 mmol), sodium hydride (14.47 mg, $0.362 \mathrm{mmol}$ ), and 3-(bromomethyl)pyridine, $\mathrm{HBr}$ (45.8 mg, 0.181 mmol), gave 4-(isopentyl(pyridin-2-ylmethyl)amino)-6-morpholino-1,3,5-triazine-2-carbonitrile (35 mg, $0.095 \mathrm{mmol}, 52.6 \%$ yield $) ;{ }^{1} \mathrm{H}$ NMR $\left(\mathrm{CDCl}_{3}, 500 \mathrm{MHz}\right) \delta 8.47$ - $8.56(\mathrm{~m}, 2 \mathrm{H}), 7.45$ - $7.64(\mathrm{~m}, 1 \mathrm{H}), 7.21$ 7.29 (m, 1H), 4.79 (s, 1H), 4.74 (s, 1H), 3.83 (br. s., 1H), 3.78 (br. s., 2H), 3.70 - 3.74 (m, 2H), 3.68 (br. s., 1H), 3.63 (br. s., $2 \mathrm{H}), 3.53$ - $3.59(\mathrm{~m}, 1 \mathrm{H}), 3.43$ - $3.49(\mathrm{~m}, 1 \mathrm{H}), 1.49$ - $1.63(\mathrm{~m}, 1 \mathrm{H}), 1.38$ - 1.49 (m, 
2H), $0.91(\mathrm{~d}, J=6.60 \mathrm{~Hz}, 3 \mathrm{H}), 0.90(\mathrm{~d}, J=6.60 \mathrm{~Hz}, 4 \mathrm{H}) ;{ }^{13} \mathrm{C} \mathrm{NMR}\left(\mathrm{CDCl}_{3}, 126 \mathrm{MHz}\right) \delta 164.2,164.1$, $163.6,163.4,152.0,151.9,149.2,149.1,149.0,148.9,135.6,134.8,133.1,133.0,123.5,115.5,115.4$, $66.7,66.6,66.4,66.3,47.6,47.5,45.7,44.9,44.0,43.9,43.4,36.3,35.5,26.0,25.9,22.5$; HRMS-ESI (m/z): $[\mathrm{M}+\mathrm{H}]^{+}$calcd for, $\mathrm{C}_{19} \mathrm{H}_{25} \mathrm{~N}_{7} \mathrm{O} ; 368.2193$; found, 368.2194

\section{4-(Isopentyl(pyridin-4-ylmethyl)amino)-6-morpholino-1,3,5-triazine-2-carbonitrile (17)}

General procedure B3 from 4-(isopentylamino)-6-morpholino-1,3,5-triazine-2-carbonitrile (100 mg, $0.362 \mathrm{mmol}$ ), sodium hydride (28.9 mg, $0.724 \mathrm{mmol}$ ), and 4-(chloromethyl)pyridine, $\mathrm{HCl}$ (59.4 mg, $0.362 \mathrm{mmol}$ ) gave 4-(isopentyl(pyridin-4-ylmethyl)amino)-6-morpholino-1,3,5-triazine-2-carbonitrile (82.4 mg, $0.224 \mathrm{mmol}, 62.0 \%$ yield); ${ }^{1} \mathrm{H} \mathrm{NMR}\left(\mathrm{CDCl}_{3}, 500 \mathrm{MHz}\right) \delta 8.48-8.59(\mathrm{~m}, 2 \mathrm{H}), 7.09(\mathrm{dd}, J=$ 4.89, $9.78 \mathrm{~Hz}, 2 \mathrm{H}), 4.78$ (s, 1H), 4.70 (s, 1H), 3.82 (br. s., 1H), 3.74 - 3.80 (m, 2H), 3.71 (br. s., 2H), 3.65 (br. s., $1 \mathrm{H}), 3.51-3.61(\mathrm{~m}, 3 \mathrm{H}), 3.41-3.50(\mathrm{~m}, 1 \mathrm{H}), 1.48-1.62(\mathrm{~m}, 1 \mathrm{H}), 1.36-1.48(\mathrm{~m}, 2 \mathrm{H}), 0.90(\mathrm{~d}, J=$ $6.85 \mathrm{~Hz}, 3 \mathrm{H}), 0.88(\mathrm{~d}, J=6.85 \mathrm{~Hz}, 3 \mathrm{H}) ;{ }^{13} \mathrm{C} \mathrm{NMR}\left(\mathrm{CDCl}_{3}, 126 \mathrm{MHz}\right) \delta 164.3,164.2,163.5,163.3,152.0$, $151.9,149.9,146.7,146.5,122.2,121.8,115.4,115.3,66.6,66.5,66.3,66.2,49.3,49.1,46.1,45.3,43.9$, 43.8, 43.4, 43.3, 36.4, 35.5, 25.9, 25.8, 22.5, 22.4; HRMS-ESI (m/z): $[\mathrm{M}+\mathrm{H}]^{+}$calcd for, $\mathrm{C}_{19} \mathrm{H}_{25} \mathrm{~N}_{7} \mathrm{O}$; 368.2193; found, 368.2195

\section{4-((4-Chlorobenzyl)(isopentyl)amino)-6-morpholino-1,3,5-triazine-2-carbonitrile (22)}

General procedure B3 from 4-(isopentylamino)-6-morpholino-1,3,5-triazine-2-carbonitrile (100 mg, $0.362 \mathrm{mmol})$, sodium hydride $(28.9 \mathrm{mg}, 0.724 \mathrm{mmol})$, and 1-(bromomethyl)-4-chlorobenzene (74.4 mg, $0.362 \mathrm{mmol}$ ) gave 4-((4-chlorobenzyl)(isopentyl)amino)-6-morpholino-1,3,5-triazine-2-carbonitrile (60.6 mg, $0.151 \mathrm{mmol}, 41.8 \%$ yield); ${ }^{1} \mathrm{H} \mathrm{NMR}\left(\mathrm{CDCl}_{3}, 500 \mathrm{MHz}\right) \delta 7.25$ - $7.32(\mathrm{~m}, 2 \mathrm{H}), 7.11$ - $7.19(\mathrm{~m}, 2 \mathrm{H})$, 4.76 (s, 1H), 4.70 (s, 1H), 3.83 (br. s., 1H), 3.79 (br. s., 2H), 3.71 - 3.75 (m, 2H), 3.69 (br. s., 1H), 3.64 (br. s., 2H), 3.53 (t, $J=7.80 \mathrm{~Hz}, 1 \mathrm{H}), 3.44(\mathrm{t}, J=7.80 \mathrm{~Hz}, 1 \mathrm{H}), 1.48-1.59(\mathrm{~m}, 1 \mathrm{H}), 1.39$ - 1.47 (m, 2H), $0.90(\mathrm{t}, J=6.60 \mathrm{~Hz}, 6 \mathrm{H}) ;{ }^{13} \mathrm{C} \mathrm{NMR}\left(\mathrm{CDCl}_{3}, 126 \mathrm{MHz}\right) \delta 164.2,164.0,163.6,163.5,152.1,151.9,135.9$, 
$135.8,133.3,133.1,131.5,129.1,128.7,128.6,115.6,115.5,66.7,66.4,49.2,45.4,44.7,44.0,43.4,36.2$ 35.4, 26.0, 25.9, 22.5; HRMS-ESI (m/z): $[\mathrm{M}+\mathrm{H}]^{+}$calcd for, $\mathrm{C}_{20} \mathrm{H}_{25} \mathrm{CIN}_{6} \mathrm{O}$; 401.1857; found, 401.1867

\section{4-((4-Acetylbenzyl)(isopentyl)amino)-6-morpholino-1,3,5-triazine-2-carbonitrile (23)}

From 4-(isopentylamino)-6-morpholino-1,3,5-triazine-2-carbonitrile (100 mg, $0.362 \mathrm{mmol})$, sodium hydride (21.71 mg, $0.543 \mathrm{mmol})$, and 1-(4-(bromomethyl)phenyl)ethan-1-one (77 mg, $0.362 \mathrm{mmol}$ ) gave 4-((4-acetylbenzyl)(isopentyl)amino)-6-morpholino-1,3,5-triazine-2-carbonitrile (31.1 mg, $0.076 \mathrm{mmol}$, $21.04 \%$ yield); ${ }^{1} \mathrm{H} \mathrm{NMR}\left(\mathrm{CDCl}_{3}, 500 \mathrm{MHz}\right) \delta 7.91(\mathrm{~d}, J=7.34 \mathrm{~Hz}, 2 \mathrm{H}), 7.23$ - $7.32(\mathrm{~m}, 2 \mathrm{H}), 4.84(\mathrm{~s}$, 1H), 4.78 (s, 1H), 3.83 (br. s., 1H), 3.79 (br. s., 1H), 3.73 (d, J=4.40 Hz, 2H), 3.64 - 3.70 (m, 2H), 3.59 $3.64(\mathrm{~m}, 2 \mathrm{H}), 3.56(\mathrm{t}, J=7.80 \mathrm{~Hz}, 1 \mathrm{H}), 3.46(\mathrm{t}, J=7.80 \mathrm{~Hz}, 1 \mathrm{H}), 2.59(\mathrm{~s}, 3 \mathrm{H}), 1.49-1.61(\mathrm{~m}, 1 \mathrm{H}), 1.38-$ $1.49(\mathrm{~m}, 2 \mathrm{H}), 0.90(\mathrm{t}, J=7.58 \mathrm{~Hz}, 6 \mathrm{H}) ;{ }^{13} \mathrm{C} \mathrm{NMR}\left(\mathrm{CDCl}_{3}, 126 \mathrm{MHz}\right) \delta 197.6,164.3,164.1,163.6,163.4$, $152.0,151.9,143.0,142.9,136.4,136.3,129.8,128.7,127.9,127.6,127.2,115.5,70.7,70.6,70.4,70.3$, $69.2,68.7,66.7,66.6,66.4,66.3,49.7,45.7,45.1,44.0,43.9,43.4,36.3,35.4,26.6,26.0,25.9,22.5$; HRMS-ESI (m/z): [M+H] $]^{+}$calcd for, $\mathrm{C}_{22} \mathrm{H}_{28} \mathrm{~N}_{6} \mathrm{O}$; 409.2347; found, 409.2350

\section{4-(isopentyl(2-methylbenzyl)amino)-6-morpholino-1,3,5-triazine-2-carbonitrile (24)}

General Procedure B3 starting from 4-(isopentylamino)-6-morpholino-1,3,5-triazine-2-carbonitrile (100 $\mathrm{mg}, 0.362 \mathrm{mmol}$ ) and 1-(bromomethyl)-2-methylbenzene (100 mg, $0.543 \mathrm{mmol})$ gave 4-(isopentyl(2methylbenzyl)amino)-6-morpholino-1,3,5-triazine-2-carbonitrile (78.7 mg, 0.207 mmol, $57.2 \%$ yield); ${ }^{1} \mathrm{H}$ NMR $\left(\mathrm{CDCl}_{3}, 500 \mathrm{MHz}\right) \delta 7.22-7.10(\mathrm{~m}, 3 \mathrm{H}), 7.08-6.98(\mathrm{~m}, 1 \mathrm{H}), 4.79(\mathrm{~d}, \mathrm{~J}=28.6 \mathrm{~Hz}, 2 \mathrm{H}), 3.91-$ $3.56(\mathrm{~m}, 8 \mathrm{H}), 3.52(\mathrm{~s}, 1 \mathrm{H}), 3.49-3.40(\mathrm{~m}, 1 \mathrm{H}), 2.30(\mathrm{~d}, \mathrm{~J}=4.5 \mathrm{~Hz}, 3 \mathrm{H}), 1.59-1.48(\mathrm{~m}, 1 \mathrm{H}), 1.48-1.37$ $(\mathrm{m}, 2 \mathrm{H}), 0.90(\mathrm{dd}, \mathrm{J}=6.6,5.3 \mathrm{~Hz}, 6 \mathrm{H}) .{ }^{13} \mathrm{C} \mathrm{NMR}\left(\mathrm{CDCl}_{3}, 126 \mathrm{MHz}\right) \delta 164.03,163.70,151.96,136.29$, $135.91,134.88,134.78,130.50,130.43,127.36,127.31,127.19,127.17,126.11,126.02,115.60,66.37$, 47.73, 47.52, 44.97, 44.64, 36.15, 35.32, 26.12, 25.98, 22.58, 22.53, 19.21. HRMS-ESI (m/z): [M+H] ${ }^{+}$ calcd for $\mathrm{C}_{21} \mathrm{H}_{28} \mathrm{~N}_{6} \mathrm{O}$, 381.2397; found, 381.2401 


\section{4-(Isopentyl(3-methylbenzyl)amino)-6-morpholino-1,3,5-triazine-2-carbonitrile (25)}

General procedure B3 from 4-(isopentylamino)-6-morpholino-1,3,5-triazine-2-carbonitrile (100 mg, $0.362 \mathrm{mmol})$, sodium hydride (21.71 $\mathrm{mg}, 0.543 \mathrm{mmol})$, and 1-(bromomethyl)-3-methylbenzene (67.0 $\mathrm{mg}$, $0.362 \mathrm{mmol}$ ) gave 4-(isopentyl(3-methylbenzyl)amino)-6-morpholino-1,3,5-triazine-2-carbonitrile (69.7 mg, $0.183 \mathrm{mmol}, 50.6 \%$ yield $) ;{ }^{1} \mathrm{H}$ NMR $\left(\mathrm{CDCl}_{3}, 500 \mathrm{MHz}\right) \delta 7.21(\mathrm{t}, J=7.58 \mathrm{~Hz}, 1 \mathrm{H}), 7.09(\mathrm{~d}, J=7.34$ Hz, 1H), 6.97 - 7.04 (m, 2H), 4.77 (s, 1H), 4.72 (s, 1H), 3.83 (br. s., 1H), 3.80 (br. s., 2H), 3.71 - 3.75 (m, 2H), 3.69 (br. s., 2H), 3.65 (br. s., 1H), $3.51-3.57$ (m, 1H), $3.43-3.48$ (m, 1H), 2.34 (d, J=2.93 Hz, $3 \mathrm{H}), 1.49$ - $1.60(\mathrm{~m}, 1 \mathrm{H}), 1.40-1.48(\mathrm{~m}, 2 \mathrm{H}), 0.91(\mathrm{t}, J=7.09 \mathrm{~Hz}, 6 \mathrm{H}) ;{ }^{13} \mathrm{C} \mathrm{NMR}\left(\mathrm{CDCl}_{3}, 126 \mathrm{MHz}\right) \delta$ 164.1, 163.9, 163.6, 163.5, 152.0, 151.9, 138.2, 137.3, 137.1, 128.5, 128.4, 128.2, 128.1, 128.0, 115.6, 66.7, 66.6, 66.4, 49.6, 49.5, 45.1, 44.5, 43.9, 43.4, 36.1, 35.3, 26.0, 25.9, 22.5, 21.4; HRMS-ESI (m/z): $[\mathrm{M}+\mathrm{H}]^{+}$calcd for, 381.2397; $\mathrm{C}_{21} \mathrm{H}_{28} \mathrm{~N}_{6} \mathrm{O}$; found, 381.2398

\section{4-(Isopentyl(4-methylbenzyl)amino)-6-morpholino-1,3,5-triazine-2-carbonitrile (26)}

General procedure B3 from 4-(isopentylamino)-6-morpholino-1,3,5-triazine-2-carbonitrile (100 mg, $0.362 \mathrm{mmol})$, sodium hydride (21.71 $\mathrm{mg}, 0.543 \mathrm{mmol})$, and 1-(bromomethyl)-4-methylbenzene (67.0 $\mathrm{mg}$, $0.362 \mathrm{mmol}$ ) gave 4-(isopentyl(4-methylbenzyl)amino)-6-morpholino-1,3,5-triazine-2-carbonitrile (88 mg, $0.231 \mathrm{mmol}, 63.9 \%$ yield); ${ }^{1} \mathrm{H} \mathrm{NMR}\left(\mathrm{CDCl}_{3}, 500 \mathrm{MHz}\right) \delta 7.07$ - $7.20(\mathrm{~m}, 4 \mathrm{H}), 4.76(\mathrm{~s}, 1 \mathrm{H}), 4.71$ (s, 1H), 3.82 (br. s., 1H), 3.79 (br. s., 2H), 3.71 - 3.74 (m, 2H), 3.69 (br. s., 2H), 3.65 (br. s., 1H), 3.49 - 3.57 (m, 1H), $3.40-3.49(\mathrm{~m}, 1 \mathrm{H}), 2.34(\mathrm{~s}, 3 \mathrm{H}), 1.50-1.59(\mathrm{~m}, 1 \mathrm{H}), 1.41-1.50(\mathrm{~m}, 2 \mathrm{H}), 0.91(\mathrm{t}, J=6.36 \mathrm{~Hz}$, $6 \mathrm{H}) ;{ }^{13} \mathrm{C} \mathrm{NMR}\left(\mathrm{CDCl}_{3}, 126 \mathrm{MHz}\right) \delta 164.1,163.9,163.6,163.5,152.0,151.9,137.1,137.0,134.2,129.2$, $127.7,127.3,115.6,66.6,66.4,60.3,49.4,49.3,45.0,44.5,43.9,43.8,43.4,36.1,35.3,26.0,25.9,22.5$, 21.1, 21.0; $[\mathrm{M}+\mathrm{H}]^{+}$calcd for, 381.2397; $\mathrm{C}_{21} \mathrm{H}_{28} \mathrm{~N}_{6} \mathrm{O}$; found, 381.2399

\section{4-((3,5-Dimethylbenzyl)(isopentyl)amino)-6-morpholino-1,3,5-triazine-2-carbonitrile (27)}

General procedure B3 from 4-(isopentylamino)-6-morpholino-1,3,5-triazine-2-carbonitrile (100 mg, $0.362 \mathrm{mmol})$, sodium hydride $(21.71 \mathrm{mg}, 0.543 \mathrm{mmol})$ and 1-(bromomethyl)-3,5-dimethylbenzene (72.0 
mg, $0.362 \mathrm{mmol})$ gave 4-((3,5-dimethylbenzyl)(isopentyl)amino)-6-morpholino-1,3,5-triazine-2carbonitrile (34.4 mg, $0.087 \mathrm{mmol}, 24.10 \%$ yield); ${ }^{1} \mathrm{H}$ NMR $\left(\mathrm{CDCl}_{3}, 500 \mathrm{MHz}\right) \delta 6.92(\mathrm{~s}, 1 \mathrm{H}), 6.83$ (s, 2H), $4.74(\mathrm{~s}, 1 \mathrm{H}), 4.69(\mathrm{~s}, 1 \mathrm{H}), 3.78-3.87(\mathrm{~m}, 3 \mathrm{H}), 3.68$ - $3.76(\mathrm{~m}, 4 \mathrm{H}), 3.62-3.68(\mathrm{~m}, 1 \mathrm{H}), 3.51-3.57$ (m, 1H), 3.42 - 3.49 (m, 1H), 2.31 (br. s., 3H), 2.30 (br. s., 3H), 1.55 (td, $J=6.66,13.57 \mathrm{~Hz}, 1 \mathrm{H}), 1.41$ $1.49(\mathrm{~m}, 2 \mathrm{H}), 0.93(\mathrm{~d}, J=7.10 \mathrm{~Hz}, 3 \mathrm{H}), 0.91(\mathrm{~d}, J=7.10 \mathrm{~Hz}, 3 \mathrm{H}) ;{ }^{13} \mathrm{C} \mathrm{NMR}\left(\mathrm{CDCl}_{3}, 126 \mathrm{MHz}\right) \delta 164.1$, 163.9, 163.6, 163.5, 152.0, 151.9, 138.1, 138.1, 137.2, 137.0, 129.1, 129.0, 125.4, 125.1, 115.6, 115.6, 66.6, 66.4, 49.4, 49.4, 45.0, 44.4, 43.9, 43.4, 36.1, 35.2, 26.0, 25.9, 22.5, 22.5, 21.3; HRMS-ESI (m/z): $[\mathrm{M}+\mathrm{H}]^{+}$calcd for, $\mathrm{C}_{22} \mathrm{H}_{30} \mathrm{~N}_{6} \mathrm{O} ; 395.2554$; found, 395.2554

\section{4-((2,3-Dimethylbenzyl)(isopentyl)amino)-6-morpholino-1,3,5-triazine-2-carbonitrile (28)}

General procedure B3 from 4-(isopentylamino)-6-morpholino-1,3,5-triazine-2-carbonitrile (100 mg, $0.362 \mathrm{mmol})$, sodium hydride (21.71 $\mathrm{mg}, 0.543 \mathrm{mmol})$, and 1-(bromomethyl)-2,3-dimethylbenzene (72.0 mg, $0.362 \mathrm{mmol})$ gave 4-((2,3-dimethylbenzyl)(isopentyl)amino)-6-morpholino-1,3,5-triazine-2carbonitrile (63.5 mg, $0.161 \mathrm{mmol}, 44.5 \%$ yield); ${ }^{1} \mathrm{H} \mathrm{NMR}\left(\mathrm{CDCl}_{3}, 500 \mathrm{MHz}\right) \delta 7.10(\mathrm{~d}, J=7.34 \mathrm{~Hz}$, $1 \mathrm{H}), 7.05(\mathrm{t}, J=7.58 \mathrm{~Hz}, 1 \mathrm{H}), 6.89(\mathrm{dd}, J=7.58,15.41 \mathrm{~Hz}, 1 \mathrm{H}), 4.82(\mathrm{~s}, 1 \mathrm{H}), 4.77(\mathrm{~s}, 1 \mathrm{H}), 3.82(\mathrm{~d}, J=$ $14.18 \mathrm{~Hz}, 3 \mathrm{H}), 3.74$ (d, $J=4.40 \mathrm{~Hz}, 2 \mathrm{H}), 3.69$ (br. s., $1 \mathrm{H}), 3.64$ (br. s., $2 \mathrm{H}), 3.45-3.50$ (m, 1H), 3.40 $3.45(\mathrm{~m}, 1 \mathrm{H}), 2.31(\mathrm{~s}, 3 \mathrm{H}), 2.18(\mathrm{~d}, J=6.36 \mathrm{~Hz}, 3 \mathrm{H}), 1.52(\mathrm{qd}, J=6.66,13.27 \mathrm{~Hz}, 1 \mathrm{H}), 1.37$ - $1.46(\mathrm{~m}$, 2H), $0.89(\mathrm{~d}, J=3.91 \mathrm{~Hz}, 3 \mathrm{H}), 0.88(\mathrm{~d}, J=3.91 \mathrm{~Hz}, 3 \mathrm{H}) ;{ }^{13} \mathrm{C} \mathrm{NMR}\left(\mathrm{CDCl}_{3}, 126 \mathrm{MHz}\right) \delta 163.9,163.7$, $163.5,152.0,151.9,137.2,137.1,134.8,134.6,134.5,129.1,125.5,125.4,125.3,125.1,115.7,115.6$, 66.7, 66.4, 48.2, 47.9, 44.6, 44.4, 43.9, 43.4, 36.0, 35.2, 26.1, 25.9, 22.5, 20.5, 20.4, 14.8; HRMS-ESI (m/z): $[\mathrm{M}+\mathrm{H}]^{+}$calcd for, $\mathrm{C}_{22} \mathrm{H}_{30} \mathrm{~N}_{6} \mathrm{O} ; 395.2554$; found, 395.2555

\section{4-(Isopentyl(3-methoxybenzyl)amino)-6-morpholino-1,3,5-triazine-2-carbonitrile (30)}

General procedure B3 from 4-(isopentylamino)-6-morpholino-1,3,5-triazine-2-carbonitrile (100 mg, $0.362 \mathrm{mmol})$, sodium hydride $(21.71 \mathrm{mg}, 0.543 \mathrm{mmol})$, and 1-(bromomethyl)-3-methoxybenzene (72.8 mg, $0.362 \mathrm{mmol}$ ) gave 4-(isopentyl(3-methoxybenzyl)amino)-6-morpholino-1,3,5-triazine-2-carbonitrile 
(70.2 mg, $0.177 \mathrm{mmol}, 48.9 \%$ yield); ${ }^{1} \mathrm{H} \mathrm{NMR}\left(\mathrm{CDCl}_{3}, 500 \mathrm{MHz}\right) \delta 7.17$ - $7.30(\mathrm{~m}, 1 \mathrm{H}), 6.74-6.83(\mathrm{~m}$, 3H), 4.77 (s, 1H), 4.71 (s, 1H), 3.82 (br. s., 1H), 3.79 (d, J=6.36 Hz, 5H), 3.70 - 3.74 (m, 2H), 3.68 (br. s., $2 \mathrm{H}), 3.63$ (br. s., 1H), 3.51 - $3.58(\mathrm{~m}, 1 \mathrm{H}), 3.42$ - $3.49(\mathrm{~m}, 1 \mathrm{H}), 1.49$ - $1.60(\mathrm{~m}, 1 \mathrm{H}), 1.40$ - 1.49 (m, 2H), $0.91(\mathrm{t}, J=6.85 \mathrm{~Hz}, 6 \mathrm{H}) ;{ }^{13} \mathrm{C} \mathrm{NMR}\left(\mathrm{CDCl}_{3}, 126 \mathrm{MHz}\right) \delta 164.1,164.0,163.6,163.5,159.8,152.0$, $151.9,139.0,138.9,129.6,129.5,120.0,119.6,115.6,115.5,113.6,113.4,112.4,112.1,66.7,66.3,55.2$, 49.6, 45.2, 44.7, 43.9, 43.8, 43.4, 36.2, 35.3, 26.0, 25.9, 22.5; HRMS-ESI (m/z): $[\mathrm{M}+\mathrm{H}]^{+}$calcd for, $\mathrm{C}_{21} \mathrm{H}_{28} \mathrm{~N}_{6} \mathrm{O}_{2} ; 397.2352$; found, 397.2356

\section{4-(Isopentyl(3-(trifluoromethyl)benzyl)amino)-6-morpholino-1,3,5-triazine-2-carbonitrile (29)}

General procedure B3 from 4-(isopentylamino)-6-morpholino-1,3,5-triazine-2-carbonitrile (100 mg, $0.362 \mathrm{mmol})$, sodium hydride $(21.71 \mathrm{mg}, 0.543 \mathrm{mmol})$, and 1-(bromomethyl)-3-(trifluoromethyl)benzene (86 mg, $0.362 \mathrm{mmol}$ ) gave 4-(isopentyl(3-(trifluoromethyl)benzyl)amino)-6-morpholino-1,3,5-triazine-2carbonitrile (119 mg, $0.274 \mathrm{mmol}, 76 \%$ yield); ${ }^{1} \mathrm{H} \mathrm{NMR}\left(\mathrm{CDCl}_{3}, 500 \mathrm{MHz}\right) \delta 7.52$ - 7.58 (m, 1H), 7.43 $7.52(\mathrm{~m}, 2 \mathrm{H}), 7.36-7.43(\mathrm{~m}, 1 \mathrm{H}), 4.85(\mathrm{~s}, 1 \mathrm{H}), 4.78(\mathrm{~s}, 1 \mathrm{H}), 3.82-3.88(\mathrm{~m}, 1 \mathrm{H}), 3.77-3.82(\mathrm{~m}, 2 \mathrm{H})$ $3.71-3.77$ (m, 2H), 3.68 (br. s., $1 \mathrm{H}), 3.56$ - $3.66(\mathrm{~m}, 3 \mathrm{H}), 3.45$ - $3.51(\mathrm{~m}, 1 \mathrm{H}), 1.50$ - 1.61 (m, $1 \mathrm{H}), 1.37$ $1.50(\mathrm{~m}, 2 \mathrm{H}), 0.92(\mathrm{~d}, J=6.36 \mathrm{~Hz}, 3 \mathrm{H}), 0.90(\mathrm{~d}, J=6.36 \mathrm{~Hz}, 3 \mathrm{H}) ;{ }^{13} \mathrm{C} \mathrm{NMR}\left(\mathrm{CDCl}_{3}, 126 \mathrm{MHz}\right) \delta 164.2$ 164.1, 163.6, 163.4, 152.0, 151.9, 138.7, 138.4, 131.0, 130.7, 130.4, 129.1, 124.3, 124.2, 124.1, 115.5, 115.4, 66.6, 66.3, 66.2, 49.7, 49.5, 45.8, 44.9, 43.9, 43.4, 43.3, 36.3, 35.4, 26.0, 25.8, 22.5, 22.4; HRMSESI (m/z): $[\mathrm{M}+\mathrm{H}]^{+}$calcd for, $\mathrm{C}_{21} \mathrm{H}_{25} \mathrm{~F}_{3} \mathrm{~N}_{6} \mathrm{O}$; 435.2115; found, 435.2095

\section{4-(Isopentyl(2-nitrobenzyl)amino)-6-morpholino-1,3,5-triazine-2-carbonitrile (31)}

General procedure B3 from 4-(isopentylamino)-6-morpholino-1,3,5-triazine-2-carbonitrile (150 mg, $0.543 \mathrm{mmol}$ ), sodium hydride (32.6 mg, $0.814 \mathrm{mmol})$, 1-(bromomethyl)-2-nitrobenzene (117 mg, 0.543 mmol) gave 4-(isopentyl(2-nitrobenzyl)amino)-6-morpholino-1,3,5-triazine-2-carbonitrile (46.8 mg, $0.114 \mathrm{mmol}, 20.95 \%$ yield); ${ }^{1} \mathrm{H}$ NMR $\left(\mathrm{CDCl}_{3}, 500 \mathrm{MHz}\right) \delta 8.01-8.13(\mathrm{~m}, 1 \mathrm{H}), 7.57(\mathrm{t}, J=7.58 \mathrm{~Hz}, 1 \mathrm{H})$, 7.39 - $7.48(\mathrm{~m}, 1 \mathrm{H}), 7.20$ (t, $J=9.30 \mathrm{~Hz}, 1 \mathrm{H}), 5.15$ (s, 1H), 5.03 (s, 1H), 3.82 (br. s., 2H), 3.73 (br. s., 
3H), 3.57 - 3.67 (m, 2H), 3.46 - 3.57 (m, 2H), 3.42 (br. s., 1H), 1.53 - 1.62 (m, 1H), 1.45 - 1.53 (m, 2H), $0.92(\mathrm{~d}, J=6.36 \mathrm{~Hz}, 3 \mathrm{H}), 0.90(\mathrm{~d}, J=6.36 \mathrm{~Hz}, 3 \mathrm{H}) ;{ }^{13} \mathrm{C} \mathrm{NMR}\left(\mathrm{CDCl}_{3}, 126 \mathrm{MHz}\right) \delta 164.4,164.3,163.5$, $163.3,152.0,151.9,148.3,148.2,133.6,133.4,133.3,128.1,128.0,127.9,127.8,125.3,125.0,115.4$, $115.2,66.6,66.5,66.3,66.1,47.8,47.7,46.5,45.9,43.9,43.8,43.4,43.2,36.4,35.5,26.0,25.9,22.5$, 22.4; HRMS-ESI (m/z): [M+H] $]^{+}$calcd for, $\mathrm{C}_{20} \mathrm{H}_{25} \mathrm{~N}_{7} \mathrm{O}_{3} ; 412.2092$; found, 412.2094

\section{4-(Isopentyl(3-nitrobenzyl)amino)-6-morpholino-1,3,5-triazine-2-carbonitrile (32)}

General procedure B3 from 4-(isopentylamino)-6-morpholino-1,3,5-triazine-2-carbonitrile (150 mg, $0.543 \mathrm{mmol})$, sodium hydride (32.6 mg, $0.814 \mathrm{mmol}$ ), and 1-(bromomethyl)-3-nitrobenzene (117 mg, $0.543 \mathrm{mmol}$ ) gave 4-(isopentyl(3-nitrobenzyl)amino)-6-morpholino-1,3,5-triazine-2-carbonitrile (43.2 mg, 0.105 mmol, $19.34 \%$ yield); ${ }^{1} \mathrm{H}$ NMR $\left(\mathrm{CDCl}_{3}, 500 \mathrm{MHz}\right) \delta 8.09$ (br. s., $\left.1 \mathrm{H}\right), 7.99-8.08$ (m, 1H), $7.56(\mathrm{t}, J=7.83 \mathrm{~Hz}, 1 \mathrm{H}), 7.49(\mathrm{t}, J=8.31 \mathrm{~Hz}, 1 \mathrm{H}), 4.86(\mathrm{~s}, 1 \mathrm{H}), 4.80(\mathrm{~s}, 1 \mathrm{H}), 3.75-3.84(\mathrm{~m}, 3 \mathrm{H}), 3.70$ (br. s., 2H), $3.54-3.66(\mathrm{~m}, 4 \mathrm{H}), 3.44-3.53(\mathrm{~m}, 1 \mathrm{H}), 1.52(\mathrm{tt}, J=6.60,12.96 \mathrm{~Hz}, 1 \mathrm{H}), 1.39$ - $1.48(\mathrm{~m}$, 2H), $0.88(\mathrm{t}, J=6.36 \mathrm{~Hz}, 6 \mathrm{H}) ;{ }^{13} \mathrm{C} \mathrm{NMR}\left(\mathrm{CDCl}_{3}, 126 \mathrm{MHz}\right) \delta 164.1,164.0,163.4,163.3,151.9,151.8$, $148.2,139.8,139.7,133.6,133.2,129.5,122.3,122.2,122.1,122.0,115.3,66.5,66.4,66.2,66.1,60.2$, 49.3, 49.2, 45.8, 45.1, 43.8, 43.3, 36.2, 35.3, 25.8, 25.7, 22.4, 22.3, 20.8; HRMS-ESI (m/z): [M+H] ${ }^{+}$ calcd for, $\mathrm{C}_{20} \mathrm{H}_{25} \mathrm{~N}_{7} \mathrm{O}_{3} ; 412.2092$; found, 412.2091

\section{4-(Isopentyl(4-nitrobenzyl)amino)-6-morpholino-1,3,5-triazine-2-carbonitrile (33)}

General procedure B3 from 4-(isopentylamino)-6-morpholino-1,3,5-triazine-2-carbonitrile (100 mg, $0.362 \mathrm{mmol})$, sodium hydride $(21.71 \mathrm{mg}, 0.543 \mathrm{mmol})$, and 1-(bromomethyl)-4-nitrobenzene (78 mg, $0.362 \mathrm{mmol}$ ) gave 4-(isopentyl(4-nitrobenzyl)amino)-6-morpholino-1,3,5-triazine-2-carbonitrile (49 mg, $0.119 \mathrm{mmol}, 32.9 \%$ yield); ${ }^{1} \mathrm{H}$ NMR $\left(\mathrm{CDCl}_{3}, 500 \mathrm{MHz}\right) \delta 8.18(\mathrm{~d}, J=7.83 \mathrm{~Hz}, 2 \mathrm{H}), 7.32-7.41(\mathrm{~m}, 2 \mathrm{H})$, 4.88 (s, 1H), 4.82 (s, 1H), 3.84 (br. s., 1H), 3.79 (br. s., 2H), 3.72 - 3.74 (m, 2H), 3.68 (br. s., 1H), 3.55 -

$3.62(\mathrm{~m}, 3 \mathrm{H}), 3.46-3.52(\mathrm{~m}, 1 \mathrm{H}), 1.52-1.60(\mathrm{~m}, 1 \mathrm{H}), 1.39-1.50(\mathrm{~m}, 2 \mathrm{H}), 0.91(\mathrm{t}, J=6.85 \mathrm{~Hz}, 6 \mathrm{H}) ;{ }^{13} \mathrm{C}$ $\mathrm{NMR}\left(\mathrm{CDCl}_{3}, 126 \mathrm{MHz}\right) \delta 164.4,164.2,163.6,163.5,152.1,152.0,147.4,147.3,145.2,145.1,128.2$, 
$127.7,123.9,115.4,66.7,66.6,66.4,66.2,49.6,46.0,45.3,44.0,43.9,43.5,43.4,36.4,35.5,26.0,25.9$, 22.5; HRMS-ESI (m/z): $[\mathrm{M}+\mathrm{H}]^{+}$calcd for, $\mathrm{C}_{20} \mathrm{H}_{25} \mathrm{~N}_{7} \mathrm{O}_{3} ; 412.2092$; found, 412.2097

\section{4-((2-Aminobenzyl)(isopentyl)amino)-6-morpholino-1,3,5-triazine-2-carbonitrile (18)}

In a round bottom flask, 4-(isopentyl(2-nitrobenzyl)amino)-6-morpholino-1,3,5-triazine-2-carbonitrile (50 $\mathrm{mg}, 0.122 \mathrm{mmol})$ was dissolved into acetone $(10 \mathrm{ml})$. Zinc dust (159 mg, $2.430 \mathrm{mmol})$ was then added, followed by the addition of aq. ammonium chloride $(0.694 \mathrm{ml}, 4.86 \mathrm{mmol})$. Reaction was left to stir for 30 min until TLC indicated starting material was consumed. Reaction was poured into water and extracted 3x with EtOAc. Combined organic layers were dried over sodium sulfate and concentrated in vacuo. Crude residue was purified by flash chromatography (0-100\% EtOAc:Hexanes gradient) to afford 4-((2-aminobenzyl) (isopentyl)amino)-6-morpholino-1,3,5-triazine-2-carbonitrile (42 mg, $0.110 \mathrm{mmol}, 91$ \% yield); ${ }^{1} \mathrm{H} \mathrm{NMR}\left(\mathrm{CDCl}_{3}, 500 \mathrm{MHz}\right) \delta 7.09-7.17(\mathrm{~m}, 1 \mathrm{H}), 7.06(\mathrm{~d}, J=7.34 \mathrm{~Hz}, 1 \mathrm{H}), 6.70(\mathrm{q}, J=7.83$ Hz, 1H), 6.65 (d, J= 7.83 Hz, 1H), 4.68 (br. s, 2H), 4.34 (br. s., 1H), 3.83 (br. s., 2H), 3.76 (br. s., 2H), 3.71 (br. s., 4H), 3.39 - 3.50 (m, 2H), 1.47 - 1.57 (m, 1H), 1.33 - 1.44 (m, 2H), 0.89 (d, J=6.36 Hz, 6H); ${ }^{13} \mathrm{C} \mathrm{NMR}\left(\mathrm{CDCl}_{3}, 126 \mathrm{MHz}\right) \delta 163.5,163.4,163.1,152.2,151.6,146.0,145.6,131.4,131.4,129.4$, 129.3, 119.7, 119.6, 117.8, 117.5, 115.7, 115.4, 115.4, 66.7, 66.4, 47.3, 47.0, 44.0, 43.8, 43.6, 43.5, 35.4, 34.7, 26.2, 26.0, 22.5, 22.5; HRMS-ESI (m/z): $[\mathrm{M}+\mathrm{H}]^{+}$calcd for, $\mathrm{C}_{20} \mathrm{H}_{27} \mathrm{~N}_{7} \mathrm{O}$; 382.2350; found, 382.2344

\section{4-((3-Aminobenzyl)(isopentyl)amino)-6-morpholino-1,3,5-triazine-2-carbonitrile (19)}

In a round bottom flask, 4-(isopentyl(3-nitrobenzyl)amino)-6-morpholino-1,3,5-triazine-2-carbonitrile (50 $\mathrm{mg}, 0.122 \mathrm{mmol})$ was dissolved into acetone $(10 \mathrm{ml})$. Zinc dust (159 mg, $2.430 \mathrm{mmol})$ was then added, followed by the addition of aq. ammonium chloride $(0.694 \mathrm{ml}, 4.86 \mathrm{mmol})$. Reaction was left to stir for 30 min until TLC indicated starting material was consumed.

Reaction was poured into water and extracted 3x with EtOAc. Combined organic layers were dried over sodium sulfate and concentrated in vacuo. Crude residue was purified by flash chromatography (0-100\% EtOAc:Hexanes gradient) to afford 4-((3-aminobenzyl) (isopentyl)amino)-6-morpholino-1,3,5-triazine-2- 
carbonitrile (32 mg, $0.084 \mathrm{mmol}, 69.0 \%$ yield); ${ }^{1} \mathrm{H} \mathrm{NMR}\left(\mathrm{CDCl}_{3}, 500 \mathrm{MHz}\right) \delta 7.03-7.16(\mathrm{~m}, 1 \mathrm{H}), 6.56$ $6.63(\mathrm{~m}, 2 \mathrm{H}), 6.54(\mathrm{~s}, 0.5 \mathrm{H}), 6.49(\mathrm{~s}, 0.5 \mathrm{H}), 4.71(\mathrm{~s}, 1 \mathrm{H}), 4.66(\mathrm{~s}, 1 \mathrm{H}), 3.82$ (br. s., $1 \mathrm{H}), 3.79$ (br. s., 2H), $3.71-3.75(\mathrm{~m}, 2 \mathrm{H}), 3.62$ - $3.71(\mathrm{~m}, 5 \mathrm{H}), 3.50$ - $3.56(\mathrm{~m}, 1 \mathrm{H}), 3.41-3.47(\mathrm{~m}, 1 \mathrm{H}), 1.48$ - $1.59(\mathrm{~m}, 1 \mathrm{H})$, 1.39 - $1.48(\mathrm{~m}, 2 \mathrm{H}), 0.91(\mathrm{t}, J=7.09 \mathrm{~Hz}, 6 \mathrm{H}) ;{ }^{13} \mathrm{C} \mathrm{NMR}\left(\mathrm{CDCl}_{3}, 126 \mathrm{MHz}\right) \delta 164.1,163.9,163.6,163.5$, $152.0,151.9,146.7,138.6,138.5,129.5,129.4,118.0,117.5,115.6,115.6,114.3,114.1,114.1,113.5$, $66.7,66.7,66.4,49.5,49.5,45.1,44.5,43.9,43.9,43.4,36.1,35.3,26.0,25.9,22.6,22.5$; HRMS-ESI (m/z): $[\mathrm{M}+\mathrm{H}]^{+}$calcd for, $\mathrm{C}_{20} \mathrm{H}_{27} \mathrm{~N}_{7} \mathrm{O} ; 382.2350$; found, 382.2348

\section{4-((4-Aminobenzyl)(isopentyl)amino)-6-morpholino-1,3,5-triazine-2-carbonitrile (20)}

In a round bottom flask, 4-(isopentyl(4-nitrobenzyl)amino)-6-morpholino-1,3,5-triazine-2-carbonitrile (100 mg, $0.243 \mathrm{mmol})$ was dissolved into acetone $(10 \mathrm{ml})$. Zinc dust (318 $\mathrm{mg}, 4.86 \mathrm{mmol})$ was then added, followed by the addition of aq. ammonium chloride $(1.389 \mathrm{ml}, 9.72 \mathrm{mmol})$. Reaction was left to stir for 30 min until TLC indicated starting material was consumed.

Reaction was poured into water and extracted $3 \mathrm{x}$ with EtOAc. Combined organic layers were dried over sodium sulfate and concentrated in vacuo. Crude residue was purified by flash chromatography (0-100\% EtOAc:Hexanes gradient) to afford 4-((4-aminobenzyl)(isopentyl) amino)-6-morpholino-1,3,5-triazine2-carbonitrile (45.6mg, $0.120 \mathrm{mmol}, 49.2 \%$ yield); ${ }^{1} \mathrm{H} \mathrm{NMR}\left(\mathrm{CDCl}_{3}, 500 \mathrm{MHz}\right) \delta 7.04(\mathrm{~d}, J=8.31 \mathrm{~Hz}$, 1H), $7.01(\mathrm{~d}, J=8.31 \mathrm{~Hz}, 1 \mathrm{H}), 6.63(\mathrm{~d}, J=7.34 \mathrm{~Hz}, 2 \mathrm{H}), 4.67$ (s, 1H), 4.63 (s, 1H), 3.80 (br. s., $2 \mathrm{H}), 3.76$ (br. s., 2H), 3.69 - 3.73 (m, 3H), 3.67 (br. s., 3H), 3.46 - 3.53 (m, 1H), 3.39 - $3.45(\mathrm{~m}, 1 \mathrm{H}), 1.47$ - 1.59 (m, 1H), $1.36-1.45(\mathrm{~m}, 2 \mathrm{H}), 0.90(\mathrm{t}, J=6.11 \mathrm{~Hz}, 6 \mathrm{H}) ;{ }^{13} \mathrm{C} \mathrm{NMR}\left(\mathrm{CDCl}_{3}, 126 \mathrm{MHz}\right) \delta 163.9,163.7,163.6$, $163.5,151.9,151.8,145.8,129.2,128.7,127.0,115.6,115.0,66.7,66.4,49.2,49.0,44.7,44.2,43.9,43.9$ 43.4, 36.1, 35.2, 26.0, 25.9, 22.5; HRMS-ESI (m/z): $[\mathrm{M}+\mathrm{H}]^{+}$calcd for, $\mathrm{C}_{20} \mathrm{H}_{27} \mathrm{~N}_{7} \mathrm{O} ; 382.2350$; found, 382.2348

4-(Isopentyl(2-(pyrrolidin-1-yl)ethyl)amino)-6-morpholino-1,3,5-triazine-2-carbonitrile (21) 
General procedure B3 from 4-(isopentylamino)-6-morpholino-1,3,5-triazine-2-carbonitrile (100 mg, $0.362 \mathrm{mmol})$, sodium hydride $(21.71 \mathrm{mg}, 0.543 \mathrm{mmol})$, and 1-(2-chloroethyl)pyrrolidine (48.4 mg, 0.362 mmol). Compound was purified by flash chromatography (1-10\% MeOH:DCM) to afford 4-(isopentyl(2-(pyrrolidin-1-yl)ethyl)amino)-6-morpholino-1,3,5-triazine-2-carbonitrile (25 mg, $0.067 \mathrm{mmol}, 18.50 \%$ yield); ${ }^{1} \mathrm{H}$ NMR $\left(\mathrm{CDCl}_{3}, 500 \mathrm{MHz}\right) \delta 3.71$ - 3.84 (m, 4H), 3.66 - 3.71 (m, 4H), 3.56 - $3.66(\mathrm{~m}, 2 \mathrm{H}), 3.45-3.56(\mathrm{~m}, 2 \mathrm{H}), 2.62(\mathrm{t}, J=7.09 \mathrm{~Hz}, 2 \mathrm{H}), 2.51-2.58(\mathrm{~m}, 4 \mathrm{H}), 1.70-1.84(\mathrm{~m}, 4 \mathrm{H})$, $1.48-1.62(\mathrm{~m}, 1 \mathrm{H}), 1.37-1.48(\mathrm{~m}, 2 \mathrm{H}), 0.91(\mathrm{t}, J=5.62 \mathrm{~Hz}, 6 \mathrm{H}) ;{ }^{13} \mathrm{C} \mathrm{NMR}\left(\mathrm{CDCl}_{3}, 126 \mathrm{MHz}\right) \delta 163.5$, 151.7, 115.6, 66.6, 66.4, 57.8, 54.4, 54.3, 54.1, 53.7, 52.9, 51.2, 46.3, 46.2, 46.1, 45.9, 43.8, 43.3, 36.6, 35.8, 26.0, 25.8, 23.4, 22.5; HRMS-ESI (m/z): $[\mathrm{M}+\mathrm{H}]^{+}$calcd for, $\mathrm{C}_{19} \mathrm{H}_{31} \mathrm{~N}_{7} \mathrm{O} ; 374.2668$; found, 374.5659

\section{4-((2-Cyanobenzyl)(isopentyl)amino)-6-morpholino-1,3,5-triazine-2-carbonitrile (34)}

General procedure B3 from 4-(isopentylamino)-6-morpholino-1,3,5-triazine-2-carbonitrile (100 mg, $0.362 \mathrm{mmol}$ ),sodium hydride (15.92 $\mathrm{mg}, 0.398 \mathrm{mmol})$, and 2-(bromomethyl)benzonitrile (70.9 mg, 0.362 mmol) gave 4-((2-cyanobenzyl)(isopentyl)amino)-6-morpholino-1,3,5-triazine-2-carbonitrile (108 mg, $0.276 \mathrm{mmol}, 76 \%$ yield); ${ }^{1} \mathrm{H}$ NMR $\left(\mathrm{CDCl}_{3}, 500 \mathrm{MHz}\right) \delta 7.64(\mathrm{~d}, J=7.34 \mathrm{~Hz}, 1 \mathrm{H}), 7.49-7.58(\mathrm{~m}, 1 \mathrm{H})$, $7.33-7.40$ (m, 1H), 7.29 (dd, J=7.83, 24.94 Hz, 1H), 5.00 (s, 1H), 4.93 (s, 1H), 3.79 (br. s., 2H), 3.74 (br. s., 1H), 3.70 (br. s., 2H), 3.53 - $3.66(\mathrm{~m}, 4 \mathrm{H}), 3.45$ - $3.53(\mathrm{~m}, 1 \mathrm{H}), 1.54(\mathrm{~s}, 1 \mathrm{H}), 1.39$ - 1.50 (m, 2H), $0.89(\mathrm{t}, J=6.85 \mathrm{~Hz}, 6 \mathrm{H}) ;{ }^{13} \mathrm{C} \mathrm{NMR}\left(\mathrm{CDCl}_{3}, 126 \mathrm{MHz}\right) \delta 164.1,163.4,163.2,151.9,151.7,141.4,141.2$, 133.0, 132.9, 132.7, 128.0, 127.8, 127.6, 127.2, 117.2, 117.1, 115.3, 115.2, 111.5, 111.1, 66.4, 66.2, 66.1, 48.1, 47.9, 46.0, 45.3, 43.8, 43.7, 43.3, 43.2, 36.2, 35.3, 25.8, 25.7, 22.3; HRMS-ESI (m/z): [M+H] ${ }^{+}$ calcd for, $\mathrm{C}_{21} \mathrm{H}_{25} \mathrm{~N}_{7} \mathrm{O}$; 392.2193; found, 392.2195

\section{4-(Isopentyl(2-(trifluoromethyl)benzyl)amino)-6-morpholino-1,3,5-triazine-2-carbonitrile (35)}

General Procedure B3 starting from 4-(isopentylamino)-6-morpholino-1,3,5-triazine-2-carbonitrile (100 $\mathrm{mg}, 0.362 \mathrm{mmol}$ ) and 1-(bromomethyl)-2-(trifluoromethyl)benzene (130 mg, $0.543 \mathrm{mmol}$ ) gave 4- 
(isopentyl(2-(trifluoromethyl)benzyl)amino)-6-morpholino-1,3,5-triazine-2-carbonitrile (89.8 mg, 0.207 mmol, $57.1 \%$ yield); ${ }^{1} \mathrm{H}$ NMR $\left(\mathrm{CDCl}_{3}, 500 \mathrm{MHz}\right) \delta 7.67(\mathrm{~d}, \mathrm{~J}=7.6 \mathrm{~Hz}, 1 \mathrm{H}), 7.55-7.45(\mathrm{~m}, 1 \mathrm{H}), 7.36$ (s, 1H), $7.19(\mathrm{~d}, \mathrm{~J}=7.8 \mathrm{~Hz}, 1 \mathrm{H}), 4.97$ (d, J = 46.9 Hz, 2H), $3.90-3.69(\mathrm{~m}, 4 \mathrm{H}), 3.69-3.37$ (m, 5H), 1.65 $-1.40(\mathrm{~m}, 3 \mathrm{H}), 1.25(\mathrm{t}, \mathrm{J}=7.1 \mathrm{~Hz}, 1 \mathrm{H}), 0.91(\mathrm{dd}, \mathrm{J}=16.1,6.5 \mathrm{~Hz}, 6 \mathrm{H}) .{ }^{13} \mathrm{C} \mathrm{NMR}\left(\mathrm{CDCl}_{3}, 126 \mathrm{MHz}\right) \delta$ $164.44,163.70,163.47,152.10,136.15,132.15,132.07,131.59,127.81,127.21,127.08,127.05,126.04$ $125.99,115.58,115.44,66.58,66.25,46.59,46.56,46.18,46.08,46.06,45.16,44.01,43.90,43.49,43.28$, 36.34, 35.33, 26.04, 25.93, 22.46, 22.44. ${ }^{19} \mathrm{~F}$ NMR (470 MHz, Chloroform-d) $\delta-58.77,-59.85,-60.32$. HRMS-ESI (m/z): $[\mathrm{M}+\mathrm{H}]^{+}$calcd for $\mathrm{C}_{21} \mathrm{H}_{25} \mathrm{~F}_{3} \mathrm{~N}_{6} \mathrm{O}$, 435.2114; found, 435.2135

\section{Methyl 2-(((4-cyano-6-morpholino-1,3,5-triazin-2-yl)(isopentyl)amino)methyl)benzoate (36)}

General Procedure B3 starting from 4-(isopentylamino)-6-morpholino-1,3,5-triazine-2-carbonitrile (250 $\mathrm{mg}, 0.905 \mathrm{mmol})$ and methyl 2-(bromomethyl)benzoate $(311 \mathrm{mg}, 1.36 \mathrm{mmol})$ gave methyl 2-(((4-cyano6-morpholino-1,3,5-triazin-2-yl)(isopentyl)amino)methyl)benzoate (41.2 mg, $0.097 \mathrm{mmol}, 10.7$ \% yield); ${ }^{1} \mathrm{H}$ NMR $\left(\mathrm{CDCl}_{3}, 500 \mathrm{MHz}\right) \delta 7.99(\mathrm{ddd}, \mathrm{J}=12.2,7.8,1.4 \mathrm{~Hz}, 1 \mathrm{H}), 7.45(\mathrm{td}, \mathrm{J}=7.6,1.4 \mathrm{~Hz}, 1 \mathrm{H}), 7.36-$ $7.28(\mathrm{~m}, 1 \mathrm{H}), 7.11(\mathrm{~d}, \mathrm{~J}=7.9 \mathrm{~Hz}, 1 \mathrm{H}), 5.22(\mathrm{~s}, 1 \mathrm{H}), 5.13(\mathrm{~s}, 1 \mathrm{H}), 3.92(\mathrm{~d}, \mathrm{~J}=4.0 \mathrm{~Hz}, 3 \mathrm{H}), 3.87-3.68$ (m, 4H), $3.68-3.40(\mathrm{~m}, 5 \mathrm{H}), 1.63-1.43(\mathrm{~m}, 3 \mathrm{H}), 1.33-1.19(\mathrm{~m}, 1 \mathrm{H}), 0.90(\mathrm{dd}, \mathrm{J}=9.0,6.4 \mathrm{~Hz}, 6 \mathrm{H}) .{ }^{13} \mathrm{C}$ $\mathrm{NMR}\left(\mathrm{CDCl}_{3}, 126 \mathrm{MHz}\right) \delta 164.33,163.67,163.49,152.00,139.49,139.48,132.49,132.44,131.14$, $130.96,128.78,128.53,126.96,126.91,126.77,126.66,115.65,66.72,66.43,66.32,52.10,48.54,48.43$, 46.21, 45.70, 43.96, 43.84, 43.45, 43.30, 36.41, 35.52, 26.12, 25.98, 22.55, 22.50. HRMS-ESI (m/z): $[\mathrm{M}+\mathrm{H}]^{+}$calcd for $\mathrm{C}_{22} \mathrm{H}_{28} \mathrm{~N}_{6} \mathrm{O}_{3}, 425.2296$; found, 425.2301

\section{4-(Isopentyl(2-methoxybenzyl)amino)-6-morpholino-1,3,5-triazine-2-carbonitrile (37)}

General Procedure B3 starting from 4-(isopentylamino)-6-morpholino-1,3,5-triazine-2-carbonitrile (100 $\mathrm{mg}, 0.362 \mathrm{mmol}$ ) and 1-(chloromethyl)-2-methoxybenzene $(85.0 \mathrm{mg}, 0.543 \mathrm{mmol})$ gave 4-(isopentyl(2methoxybenzyl)amino)-6-morpholino-1,3,5-triazine-2-carbonitrile (47.8 mg, $0.121 \mathrm{mmol}, 33.3 \%$ yield); ${ }^{1} \mathrm{H} \mathrm{NMR}\left(\mathrm{CDCl}_{3}, 500 \mathrm{MHz}\right) \delta 7.29-7.16(\mathrm{~m}, 1 \mathrm{H}), 7.16-7.04(\mathrm{~m}, 1 \mathrm{H}), 6.96-6.81(\mathrm{~m}, 2 \mathrm{H}), 4.80(\mathrm{~d}, \mathrm{~J}=$ 
$24.9 \mathrm{~Hz}, 2 \mathrm{H}), 3.85(\mathrm{~s}, 3 \mathrm{H}), 3.83-3.59(\mathrm{~m}, 7 \mathrm{H}), 3.59-3.45(\mathrm{~m}, 2 \mathrm{H}), 1.60-1.51(\mathrm{~m}, 1 \mathrm{H}), 1.50-1.43(\mathrm{~m}$, 2H), $0.92(\mathrm{dd}, \mathrm{J}=6.5,4.4 \mathrm{~Hz}, 6 \mathrm{H}) .{ }^{13} \mathrm{C} \mathrm{NMR}\left(\mathrm{CDCl}_{3}, 126 \mathrm{MHz}\right) \delta 164.11,163.66,157.40,128.51$, $128.46,128.34,128.07,125.40,125.22,120.52,120.48,115.69,110.26,110.23,55.29,45.54,45.19$, 44.55, 44.34, 36.34, 35.52, 26.13, 25.97, 22.60, 22.55. HRMS-ESI (m/z): $[\mathrm{M}+\mathrm{H}]^{+}$calcd for $\mathrm{C}_{21} \mathrm{H}_{28} \mathrm{~N}_{6} \mathrm{O}_{2}$, 397.2346; found, 397.2379

\section{4-((4-Fluorobenzyl)(propyl)amino)-6-morpholino-1,3,5-triazine-2-carbonitrile (38)}

In a dry reaction vial, 4-((4-fluorobenzyl)amino)-6-morpholino-1,3,5-triazine-2-carbonitrile (50 mg, $0.159 \mathrm{mmol})$ was dissolved into DMF (1 ml). Cesium carbonate $(104 \mathrm{mg}, 0.318 \mathrm{mmol})$ and 1 bromopropane $(0.029 \mathrm{ml}, 0.318 \mathrm{mmol})$ were added to the solution and the reaction was left to stir at $80^{\circ} \mathrm{C}$ overnight. Reaction was cooled to room temperature, poured into EtOAc and washed 3x with brine. Organic layers were combined, dried over sodium sulfate, and concentrated. Crude residue was purified by flash chromatography (0-100\% EtOAc:Hexanes gradient) to afford 4-((4-fluorobenzyl)(propyl)amino)6-morpholino-1,3,5-triazine-2-carbonitrile (24 mg, $0.067 \mathrm{mmol}, 42.3 \%$ yield); ${ }^{1} \mathrm{H} \mathrm{NMR}\left(\mathrm{CDCl}_{3}, 500\right.$ MHz) $\delta 7.11$ - 7.25 (m, 2H), 6.97 - 7.04 (m, 2H), 4.77 (s, 1H), 4.72 (s, 1H), 3.82 (br. s., 1H), 3.77 (br. s., 1H), 3.71 - $3.74(\mathrm{~m}, 2 \mathrm{H}), 3.62$ - $3.71(\mathrm{~m}, 3 \mathrm{H}), 3.44$ - $3.51(\mathrm{~m}, 1 \mathrm{H}), 3.35$ - $3.43(\mathrm{~m}, 1 \mathrm{H}), 1.53$ - $1.59(\mathrm{~m}$, 2H), $0.85-0.91(\mathrm{~m}, 3 \mathrm{H}) ;{ }^{13} \mathrm{C} \mathrm{NMR}\left(\mathrm{CDCl}_{3}, 126 \mathrm{MHz}\right) \delta 164.2,164.1,163.6,163.5,163.1,163.0,161.2$, $161.1,152.0,151.9,133.1,129.4,128.9,128.8,115.6,115.5,115.3,68.2,66.6,66.4,66.3,49.3,49.2$, 48.5, 48.0, 43.9, 43.7, 43.4, 20.7, 20.1, 11.5, 11.2, 10.4; HRMS-ESI (m/z): $[\mathrm{M}+\mathrm{H}]^{+}$calcd for, $\mathrm{C}_{18} \mathrm{H}_{21} \mathrm{FN}_{6} \mathrm{O}$; 357.1834; found, 357.1834

\section{4-(Butyl(4-fluorobenzyl)amino)-6-morpholino-1,3,5-triazine-2-carbonitrile (39)}

General procedure B3 from 4-((4-fluorobenzyl)amino)-6-morpholino-1,3,5-triazine-2-carbonitrile (100 mg, $0.318 \mathrm{mmol}$ ), sodium hydride (19.09 mg, $0.477 \mathrm{mmol}$ ) and 1-bromobutane (43.6 mg, $0.318 \mathrm{mmol})$ gave 4-(butyl(4-fluorobenzyl)amino)-6-morpholino-1,3,5-triazine-2-carbonitrile (97 mg, $0.262 \mathrm{mmol}, 82$ \% yield); ${ }^{1} \mathrm{H} \mathrm{NMR}\left(\mathrm{CDCl}_{3}, 500 \mathrm{MHz}\right) \delta 7.14$ - 7.25 (m, 2H), 6.99 (t, $\left.J=8.56 \mathrm{~Hz}, 2 \mathrm{H}\right), 4.76(\mathrm{~s}, 1 \mathrm{H}), 4.72$ 
(s, 1H), $3.75-3.88(\mathrm{~m}, 3 \mathrm{H}), 3.59-3.75(\mathrm{~m}, 5 \mathrm{H}), 3.51(\mathrm{t}, J=7.58 \mathrm{~Hz}, 1 \mathrm{H}), 3.43(\mathrm{t}, J=7.58 \mathrm{~Hz}, 1 \mathrm{H}), 1.53$

$(\mathrm{t}, J=7.34 \mathrm{~Hz}, 2 \mathrm{H}), 1.23-1.37(\mathrm{~m}, 2 \mathrm{H}), 0.86-0.97(\mathrm{~m}, 3 \mathrm{H}) ;{ }^{13} \mathrm{C} \mathrm{NMR}\left(\mathrm{CDCl}_{3}, 126 \mathrm{MHz}\right) \delta 164.2$, 164.0, 163.6, 163.4, 163.1, 163.0, 161.2, 161.0, 152.0, 151.9, 133.1, 129.4, 129.3, 128.9, 128.8, 115.5, $115.4,115.3,115.2,66.6,66.3,49.1,46.5,45.9,43.9,43.4,29.5,28.8,20.1,19.9,13.8,13.7 ;{ }^{19} \mathrm{~F}$ NMR $\left(\mathrm{CDCl}_{3}, 470 \mathrm{MHz}\right) \delta$-115.01; HRMS-ESI (m/z): [M+H] $]^{+}$calcd for, $\mathrm{C}_{19} \mathrm{H}_{23} \mathrm{FN}_{6} \mathrm{O} ; 371.1990$; found, 371.1989

\section{4-(But-3-en-1-yl(4-fluorobenzyl)amino)-6-morpholino-1,3,5-triazine-2-carbonitrile (40)}

In a dry reaction vessel, 4-((4-fluorobenzyl)amino)-6-morpholino-1,3,5-triazine-2-carbonitrile (50 mg, $0.159 \mathrm{mmol})$ was dissolved into DMF $(1 \mathrm{ml})$. Potassium carbonate $(44.0 \mathrm{mg}, 0.318 \mathrm{mmol})$ and $4-$ bromobut-1-ene (43.0 $\mathrm{mg}, 0.318 \mathrm{mmol})$ were added to the solution and the reaction was left to stir at $80^{\circ} \mathrm{C}$ overnight. Reaction was cooled to room temperature, poured into EtOAc and washed $3 \mathrm{x}$ with brine. Organic layers were combined, dried over sodium sulfate, and concentrated. Crude residue was purified by flash chromatography (0-100\% EtOAc:Hexanes gradient) to afford 4-(but-3-en-1-yl(4fluorobenzyl)amino)-6-morpholino-1,3,5-triazine-2-carbonitrile (25 mg, $0.068 \mathrm{mmol}, 42.7 \%$ yield); ${ }^{1} \mathrm{H}$ NMR $\left(\mathrm{CDCl}_{3}, 500 \mathrm{MHz}\right) \delta 7.15$ - $7.24(\mathrm{~m}, 2 \mathrm{H}), 6.97$ - $7.04(\mathrm{~m}, 2 \mathrm{H}), 5.66$ - $5.84(\mathrm{~m}, 1 \mathrm{H}), 5.00$ - 5.09 (m, 2H), $4.78(\mathrm{~s}, 1 \mathrm{H}), 4.73(\mathrm{~s}, 1 \mathrm{H}), 3.75-3.87(\mathrm{~m}, 3 \mathrm{H}), 3.63-3.75(\mathrm{~m}, 5 \mathrm{H}), 3.59(\mathrm{t}, J=7.34 \mathrm{~Hz}, 1 \mathrm{H}), 3.50$ (t, $J=7.34 \mathrm{~Hz}, 1 \mathrm{H}), 2.25-2.36(\mathrm{~m}, 2 \mathrm{H}) ;{ }^{13} \mathrm{C} \mathrm{NMR}\left(\mathrm{CDCl}_{3}, 126 \mathrm{MHz}\right) \delta 164.1,163.5,152.0,143.6,135.0$, $134.9,133.0,129.5,129.0,128.9,117.1,116.9,115.6,115.4,49.6,49.5,46.4,46.0,44.0,43.9,43.4,32.0$ 31.3; HRMS-ESI (m/z): $[\mathrm{M}+\mathrm{H}]^{+}$calcd for, $\mathrm{C}_{19} \mathrm{H}_{21} \mathrm{FN}_{6} \mathrm{O}$; 369.1834; found, 369.1842

\section{4-((4-Fluorobenzyl)(3-methylbut-3-en-1-yl)amino)-6-morpholino-1,3,5-triazine-2-carbonitrile (41)}

General procedure B3 from 4-((4-fluorobenzyl)amino)-6-morpholino-1,3,5-triazine-2-carbonitrile (100 mg, $0.318 \mathrm{mmol})$, sodium hydride (19.09 mg, $0.477 \mathrm{mmol})$, and 3-methylbut-3-en-1-yl 4methylbenzenesulfonate (76 mg, $0.318 \mathrm{mmol}$ ) gave 4-((4-fluorobenzyl)(3-methylbut-3-en-1-yl)amino)-6morpholino-1,3,5-triazine-2-carbonitrile (50.3 mg, $0.132 \mathrm{mmol}, 41.3 \%$ yield); ${ }^{1} \mathrm{H} \mathrm{NMR}\left(\mathrm{CDCl}_{3}, 500\right.$ 
MHz) $\delta 7.14$ - 7.25 (m, 2H), 6.95 - 7.07 (m, 2H), 4.77 (br. s., 2H), 4.73 (s, 1H), 4.66 (br. s., 1H), 3.76 $3.86(\mathrm{~m}, 3 \mathrm{H}), 3.72$ (br. s., $3 \mathrm{H}), 3.59-3.69$ (m, 3H), 3.54 (t, $J=7.58 \mathrm{~Hz}, 1 \mathrm{H}), 2.24(\mathrm{q}, J=7.83 \mathrm{~Hz}, 2 \mathrm{H})$, $1.74(\mathrm{~d}, J=21.52 \mathrm{~Hz}, 3 \mathrm{H}) ;{ }^{13} \mathrm{C} \mathrm{NMR}\left(\mathrm{CDCl}_{3}, 126 \mathrm{MHz}\right) \delta 164.2,164.0,163.6,163.5,163.2,163.1$, $161.3,152.0,142.6,142.5,133.0,129.5,129.5,129.0,128.9,115.6,115.5,115.4,112.2,66.7,66.4,66.3$, 49.4, 49.2, 45.4, 45.2, 44.0, 43.9, 43.4, 35.6, 34.8, 22.5, 22.4; HRMS-ESI (m/z): $[\mathrm{M}+\mathrm{H}]^{+}$calcd for, $\mathrm{C}_{20} \mathrm{H}_{23} \mathrm{FN}_{6} \mathrm{O}$; 383.1990; found, 383.1989

\section{4-((4-Fluorobenzyl)(isobutyl)amino)-6-morpholino-1,3,5-triazine-2-carbonitrile (42)}

General procedure A4 from 4-chloro-N-(4-fluorobenzyl)-N-isobutyl-6-morpholino-1,3,5-triazin-2-amine (65 mg, $0.171 \mathrm{mmol}), \mathrm{DABCO}(38.4 \mathrm{mg}, 0.342 \mathrm{mmol})$, and potassium cyanide (12.26 mg, $0.188 \mathrm{mmol})$ gave 4-((4-fluorobenzyl)(isobutyl)amino)-6-morpholino-1,3,5-triazine-2-carbonitrile (48 mg, 0.130 mmol, $76 \%$ yield); ${ }^{1} \mathrm{H}$ NMR $\left(\mathrm{CDCl}_{3}, 500 \mathrm{MHz}\right) \delta 7.08-7.23(\mathrm{~m}, 2 \mathrm{H}), 6.98(\mathrm{t}, J=8.31 \mathrm{~Hz}, 2 \mathrm{H}), 4.78(\mathrm{~s}$, 1H), 4.73 (s, 1H), 3.77 (br. s., 3H), 3.70 (br. s., 3H), 3.63 (br. s., 2H), 3.37 (d, $J=7.34 \mathrm{~Hz}, 1 \mathrm{H}), 3.27$ (d, $J$ $=7.34 \mathrm{~Hz}, 1 \mathrm{H}), 2.05-2.14(\mathrm{~m}, 1 \mathrm{H}), 0.83-0.94(\mathrm{~m}, 6 \mathrm{H}) ;{ }^{13} \mathrm{C} \mathrm{NMR}\left(\mathrm{CDCl}_{3}, 126 \mathrm{MHz}\right) \delta 164.6,164.6$, $163.4,161.1,151.9,151.9,133.0,132.9,129.4,129.3,128.8,128.8,115.5,115.5,115.4,115.3,115.3$, $66.6,66.4,66.3,53.8,53.4,49.7,49.6,46.5,43.8,43.3,37.1,36.6,31.5,27.0,26.7,21.0,20.3,20.0,20.0$, 14.8, 14.1, 14.0, 13.9; HRMS-ESI (m/z): $[\mathrm{M}+\mathrm{H}]^{+}$calcd for, $\mathrm{C}_{19} \mathrm{H}_{23} \mathrm{FN}_{6} \mathrm{O} ; 371.1996$; found, 371.2060

\section{4-((4-Fluorobenzyl)(4-methylpentyl)amino)-6-morpholino-1,3,5-triazine-2-carbonitrile (43)}

General Procedure B3 starting from 4-((4-fluorobenzyl)amino)-6-morpholino-1,3,5-triazine-2-carbonitrile (70.0 mg, $0.223 \mathrm{mmol})$ and 1-bromo-4-methylpentane $(55.1 \mathrm{mg}, 0.334 \mathrm{mmol})$ gave 4-((4fluorobenzyl)(4-methylpentyl)amino)-6-morpholino-1,3,5-triazine-2-carbonitrile (48.0 mg, 0.120 mmol, $54.1 \%$ yield); ${ }^{1} \mathrm{H} \mathrm{NMR}\left(\mathrm{CDCl}_{3}, 500 \mathrm{MHz}\right) \delta$ 7.14-7.24 (m, 2H), $7.00(\mathrm{t}, \mathrm{J}=8.61 \mathrm{~Hz}, 2 \mathrm{H}), 4.76(\mathrm{~s}, 1 \mathrm{H})$, $4.72(\mathrm{~s}, 1 \mathrm{H}), 3.74-3.86(\mathrm{~m}, 3 \mathrm{H}), 3.61-3.74(\mathrm{~m}, 5 \mathrm{H}), 3.48(\mathrm{t}, \mathrm{J}=7.43 \mathrm{~Hz}, 1 \mathrm{H}), 3.39$ (t, J = 7.63 Hz, 1H), $1.46-1.60(\mathrm{~m}, 3 \mathrm{H}), 1.05-1.19(\mathrm{~m}, 2 \mathrm{H}), 0.86(\mathrm{~d}, \mathrm{~J}=6.65 \mathrm{~Hz}, 3 \mathrm{H}), 0.84(\mathrm{~d}, \mathrm{~J}=6.70 \mathrm{~Hz}, 3 \mathrm{H}) .{ }^{13} \mathrm{C} \mathrm{NMR}$ $\left(\mathrm{CDCl}_{3}, 126 \mathrm{MHz}\right) \delta 164.2,164.0,163.6,163.5,163.4,163.3,152.0,151.9,133.1,129.7,129.6,129.2$ 
$128.7,128.7,115.7,115.6,115.6,115.5,115.4,115.1,66.7,66.4,49.6,49.4,49.3,49.1,47.0,46.8,46.7$, $43.9,43.4,36.3,36.1,35.9,35.7,35.5,27.7,27.6,27.5,25.2,24.4,22.7,22.5,22.4,22.2 .{ }^{19} \mathrm{~F}$ NMR $(376$ $\mathrm{MHz}$, Chloroform-d) $\delta$-115.07, -114.99. HRMS-ESI (m/z): $[\mathrm{M}+\mathrm{H}]^{+}$calcd for $\mathrm{C}_{21} \mathrm{H}_{27} \mathrm{FN}_{6} \mathrm{O}, 399.2303$; found, 399.2302

\section{4-((Cyclopropylmethyl)(4-fluorobenzyl)amino)-6-morpholino-1,3,5-triazine-2-carbonitrile (57)}

In a dry reaction vessel, 4-((4-fluorobenzyl)amino)-6-morpholino-1,3,5-triazine-2-carbonitrile (50 mg, $0.159 \mathrm{mmol})$ was dissolved into DMF $(0.5 \mathrm{ml})$. Potassium carbonate $(44.0 \mathrm{mg}, 0.318 \mathrm{mmol})$ and (bromomethyl)cyclopropane $(0.031 \mathrm{ml}, 0.318 \mathrm{mmol})$ were added to the solution and the reaction was left to stir at $80^{\circ} \mathrm{C}$ overnight. Reaction was cooled to room temperature, poured into EtOAc and washed $3 \mathrm{x}$ with brine. Organic layers were combined, dried over sodium sulfate, and concentrated. Crude residue was purified by flash chromatography (0-100\% EtOAc:Hexanes gradient) to afford 4((cyclopropylmethyl)(4-fluorobenzyl)amino)-6-morpholino-1,3,5-triazine-2-carbonitrile (15 mg, 0.041 mmol, $25.6 \%$ yield); ${ }^{1} \mathrm{H}$ NMR $\left(\mathrm{CDCl}_{3}, 500 \mathrm{MHz}\right) \delta 7.15-7.25(\mathrm{~m}, 2 \mathrm{H}), 7.01(\mathrm{t}, J=8.56 \mathrm{~Hz}, 2 \mathrm{H}), 4.89$ $(\mathrm{s}, 1 \mathrm{H}), 4.85(\mathrm{~s}, 1 \mathrm{H}), 3.63-3.86(\mathrm{~m}, 8 \mathrm{H}), 3.46(\mathrm{~d}, J=6.85 \mathrm{~Hz}, 1 \mathrm{H}), 3.37(\mathrm{~d}, J=6.85 \mathrm{~Hz}, 1 \mathrm{H}), 0.98-1.08$ $(\mathrm{m}, 1 \mathrm{H}), 0.49(\mathrm{dd}, J=5.38,12.72 \mathrm{~Hz}, 2 \mathrm{H}), 0.25(\mathrm{q}, J=4.89 \mathrm{~Hz}, 1 \mathrm{H}), 0.19(\mathrm{q}, J=4.89 \mathrm{~Hz}, 1 \mathrm{H}) ;{ }^{13} \mathrm{C} \mathrm{NMR}$ $\left(\mathrm{CDCl}_{3}, 126 \mathrm{MHz}\right) \delta 164.3,163.6,163.5,163.1,163.0,161.2,161.0,152.0,133.2,133.1,129.3,129.2$ $128.8,128.7,115.5,115.4,115.3,115.2,115.0,71.5,66.6,66.4,66.3,50.9,50.3,49.2,49.1,43.9,43.7$, 43.4, 14.2, 9.6, 9.1, 3.6; HRMS-ESI (m/z): $[\mathrm{M}+\mathrm{H}]^{+}$calcd for, $\mathrm{C}_{19} \mathrm{H}_{21} \mathrm{FN}_{6} \mathrm{O} ; 369.1834$; found, 369.1835

\section{4-((Cyclobutylmethyl)(4-fluorobenzyl)amino)-6-morpholino-1,3,5-triazine-2-carbonitrile (58)}

In a dry reaction vial, 4-((4-fluorobenzyl)amino)-6-morpholino-1,3,5-triazine-2-carbonitrile (50 mg, $0.159 \mathrm{mmol})$ was dissolved into DMF $(1 \mathrm{ml})$. Potassium carbonate $(44.0 \mathrm{mg}, 0.318 \mathrm{mmol})$ and (bromomethyl)cyclobutane $(47.4 \mathrm{mg}, 0.318 \mathrm{mmol})$ were added to the solution and the reaction was left to stir at $80^{\circ} \mathrm{C}$ overnight. Reaction was cooled to room temperature, poured into EtOAc and washed 3x with brine. Organic layers were combined, dried over sodium sulfate, and concentrated. Crude residue was 
purified by flash chromatography (0-100\% EtOAc:Hexanes) to afford 4-((cyclobutylmethyl)(4fluorobenzyl)amino)-6-morpholino-1,3,5-triazine-2-carbonitrile (17 mg, $0.044 \mathrm{mmol}, 27.9 \%$ yield); ${ }^{1} \mathrm{H}$ NMR $\left(\mathrm{CDCl}_{3}, 500 \mathrm{MHz}\right) \delta 7.10-7.22(\mathrm{~m}, 2 \mathrm{H}), 7.00(\mathrm{t}, J=7.83 \mathrm{~Hz}, 2 \mathrm{H}), 4.76(\mathrm{~s}, 1 \mathrm{H}), 4.71(\mathrm{~s}, 1 \mathrm{H}), 3.80$ (br. s., 3H), 3.73 (br. s., 2H), 3.69 (br. s., 1H), 3.64 (br. s., 2H), 3.60 (d, $J=7.34 \mathrm{~Hz}, 1 \mathrm{H}), 3.51$ (d, $J=$ $7.34 \mathrm{~Hz}, 1 \mathrm{H}), 2.53$ - 2.69 (m, 1H), 1.97 (br. s., $2 \mathrm{H}), 1.79$ - $1.92(\mathrm{~m}, 2 \mathrm{H}), 1.64-1.79(\mathrm{~m}, 2 \mathrm{H}) ;{ }^{13} \mathrm{C} \mathrm{NMR}$ $\left(\mathrm{CDCl}_{3}, 126 \mathrm{MHz}\right) \delta 164.5,164.4,163.6,163.5,163.2,161.2,161.1,152.0,151.9,133.2,129.3,129.3$, $128.7,128.7,115.6,115.5,115.3,66.6,66.4,66.3,51.7,51.1,49.5,43.9,43.4,34.3,34.0,26.8,26.5$, 18.6, 18.5; HRMS-ESI (m/z): [M+H] $]^{+}$calcd for, $\mathrm{C}_{20} \mathrm{H}_{23} \mathrm{FN}_{6} \mathrm{O} ; 383.1990$; found, 383.1994

\section{4-((Cyclopentylmethyl)(4-fluorobenzyl)amino)-6-morpholino-1,3,5-triazine-2-carbonitrile (59)}

General procedure B3 from 4-((4-fluorobenzyl)amino)-6-morpholino-1,3,5-triazine-2-carbonitrile (100 $\mathrm{mg}, 0.318 \mathrm{mmol})$, sodium hydride $(14.00 \mathrm{mg}, 0.350 \mathrm{mmol})$, and (bromomethyl)cyclopentane $(0.039 \mathrm{ml}$, $0.318 \mathrm{mmol}$ ) gave 4-((cyclopentylmethyl)(4-fluorobenzyl)amino)-6-morpholino-1,3,5-triazine-2carbonitrile (31.6 mg, $0.080 \mathrm{mmol}, 25.05 \%$ yield); ${ }^{1} \mathrm{H} \mathrm{NMR}\left(\mathrm{CDCl}_{3}, 500 \mathrm{MHz}\right) \delta 1.22(\mathrm{~m}) 1.54(\mathrm{~m}) 1.64$ (m) $2.27(\mathrm{~m}) 3.42$ (d) 3.51 (d) $3.66(\mathrm{~m}) 3.72(\mathrm{~m}) 3.79$ (m) 4.76 (s) 4.81 (s) 7.00 (t) $7.18(\mathrm{~m})$; ${ }^{13} \mathrm{C}$ NMR $\left(\mathrm{CDCl}_{3}, 126 \mathrm{MHz}\right) \delta 164.5,163.5,163.1,163.0,161.2,161.0,151.9,133.1,133.0,129.4,129.3,128.8$ $128.7,115.6,115.5,115.3,66.7,66.4,66.3,50.9,50.3,49.3,49.2,43.9,43.4,38.7,38.2,30.5,30.3,24.9$, 24.8; ${ }^{19} \mathrm{~F}$ NMR $\left(\mathrm{CDCl}_{3}, 470 \mathrm{MHz}\right) \delta$-115.16 (m); HRMS-ESI (m/z): [M+H] $]^{+}$calcd for, $\mathrm{C}_{21} \mathrm{H}_{25} \mathrm{FN}_{6} \mathrm{O}$; 397.2147; found, 397.2141

\section{4-((Cyclohexylmethyl)(4-fluorobenzyl)amino)-6-morpholino-1,3,5-triazine-2-carbonitrile (56)}

General procedure B3 from 4-((4-fluorobenzyl)amino)-6-morpholino-1,3,5-triazine-2-carbonitrile (100 $\mathrm{mg}, 0.318 \mathrm{mmol}$, sodium hydride $(15.27 \mathrm{mg}, 0.382 \mathrm{mmol})$, and (bromomethyl)cyclohexane $(0.043 \mathrm{ml}$, $0.318 \mathrm{mmol}$ ) gave 4-((cyclohexylmethyl)(4-fluorobenzyl)amino)-6-morpholino-1,3,5-triazine-2carbonitrile (105 mg, $0.256 \mathrm{mmol}, 80 \%$ yield); ${ }^{1} \mathrm{H}$ NMR $\left(\mathrm{CDCl}_{3}, 500 \mathrm{MHz}\right) \delta 7.11$ - 7.22 (m, 2H), 6.92 7.05 (m, 2H), 4.79 (s, 1H), 4.73 (s, 1H), 3.78 - 3.87 (m, 2H), 3.76 (br. s., 1H), 3.71 - 3.75 (m, 2H), 3.60 - 
$3.70(\mathrm{~m}, 3 \mathrm{H}), 3.40(\mathrm{~d}, J=7.83 \mathrm{~Hz}, 1 \mathrm{H}), 3.30(\mathrm{~d}, J=7.34 \mathrm{~Hz}, 1 \mathrm{H}), 1.68$ - $1.83(\mathrm{~m}, 3 \mathrm{H}), 1.57$ - $1.68(\mathrm{~m}$, 3H), 1.15 - $1.24(\mathrm{~m}, 3 \mathrm{H}), 0.88$ - $1.04(\mathrm{~m}, 2 \mathrm{H}) ;{ }^{13} \mathrm{C} \mathrm{NMR}\left(\mathrm{CDCl}_{3}, 126 \mathrm{MHz}\right) \delta 164.7,164.6,164.1,163.8$, $163.5,161.2,161.1,152.0,151.9,133.1,133.0,129.4,128.8,115.6,115.5,115.4,115.3,66.7,66.4,66.3$, 52.6, 52.2, 49.8, 49.7, 44.0, 43.4, 36.5, 36.3, 31.2, 30.7, 26.3, 25.8; HRMS-ESI (m/z): $[\mathrm{M}+\mathrm{H}]^{+}$calcd for, $\mathrm{C}_{22} \mathrm{H}_{27} \mathrm{FN}_{6} \mathrm{O}$; 411.2303; found, 411.2300

\section{4-((2-Cyclopropylethyl)(4-fluorobenzyl)amino)-6-morpholino-1,3,5-triazine-2-carbonitrile (51)}

General procedure B3 from 4-((4-fluorobenzyl)amino)-6-morpholino-1,3,5-triazine-2-carbonitrile (100 $\mathrm{mg}, 0.318 \mathrm{mmol})$, sodium hydride (19.09 $\mathrm{mg}, 0.477 \mathrm{mmol})$, and 2-cyclopropylethyl 4methylbenzenesulfonate (76 mg, $0.318 \mathrm{mmol}$ ) gave 4-((2-cyclopropylethyl)(4-fluorobenzyl)amino)-6morpholino-1,3,5-triazine-2-carbonitrile (58 mg, $0.152 \mathrm{mmol}, 47.7 \%$ yield); ${ }^{1} \mathrm{H}$ NMR $\left(\mathrm{CDCl}_{3}, 500 \mathrm{MHz}\right)$ $\delta 7.13-7.26(\mathrm{~m}, 2 \mathrm{H}), 7.00(\mathrm{t}, J=8.31 \mathrm{~Hz}, 2 \mathrm{H}), 4.78(\mathrm{~s}, 1 \mathrm{H}), 4.73(\mathrm{~s}, 1 \mathrm{H}), 3.75-3.85(\mathrm{~m}, 3 \mathrm{H}), 3.70-3.74$ (m, 2H), 3.68 (br. s., 2H), 3.65 (br. s., $1 \mathrm{H}), 3.59$ (t, $J=7.40 \mathrm{~Hz}, 1 \mathrm{H}), 3.51$ (t, $J=7.34 \mathrm{~Hz}, 1 \mathrm{H}), 1.40-1.51$ $(\mathrm{m}, 2 \mathrm{H}), 0.52-0.69(\mathrm{~m}, 1 \mathrm{H}), 0.43(\mathrm{~d}, J=7.83 \mathrm{~Hz}, 2 \mathrm{H}), 0.02-0.07(\mathrm{~m}, 1 \mathrm{H}),-0.02-0.02(\mathrm{~m}, 1 \mathrm{H}) ;{ }^{13} \mathrm{C}$ $\mathrm{NMR}\left(\mathrm{CDCl}_{3}, 126 \mathrm{MHz}\right) \delta 164.1,164.0,163.6,163.5,163.0,161.2,161.1,152.0,151.9,133.1,133.1$, $129.5,129.4,128.9,115.6,115.5,115.3,66.7,66.6,66.4,66.3,49.5,47.1,46.4,43.9,43.4,32.5,31.8$, 8.5, 8.4, 4.4, 4.3; HRMS-ESI (m/z): $[\mathrm{M}+\mathrm{H}]^{+}$calcd for, $\mathrm{C}_{20} \mathrm{H}_{23} \mathrm{FN}_{6} \mathrm{O}$; 383.1990; found, 383.1986

\section{4-((3,3-Dimethylbutyl)(4-fluorobenzyl)amino)-6-morpholino-1,3,5-triazine-2-carbonitrile (53)}

General procedure B3 from 4-((4-fluorobenzyl)amino)-6-morpholino-1,3,5-triazine-2-carbonitrile (100 $\mathrm{mg}, 0.318 \mathrm{mmol})$, sodium hydride $(19.09 \mathrm{mg}, 0.477 \mathrm{mmol})$, and 1-bromo-3,3-dimethylbutane (0.068 $\mathrm{ml}$, 0.477 mmol) gave 4-((3,3-dimethylbutyl)(4-fluorobenzyl)amino)-6-morpholino-1,3,5-triazine-2carbonitrile (26 mg, $0.065 \mathrm{mmol}, 20.51 \%$ yield); ${ }^{1} \mathrm{H} \mathrm{NMR}\left(\mathrm{CDCl}_{3}, 500 \mathrm{MHz}\right) \delta 7.15-7.25(\mathrm{~m}, 2 \mathrm{H}), 6.98$ - $7.04(\mathrm{~m}, 2 \mathrm{H}), 4.74(\mathrm{~s}, 1 \mathrm{H}), 4.69(\mathrm{~s}, 1 \mathrm{H}), 3.76$ - $3.86(\mathrm{~m}, 3 \mathrm{H}), 3.72$ (br. s., 3H), 3.63 - 3.70 (m, 2H), 3.47 $3.53(\mathrm{~m}, 1 \mathrm{H}), 3.39-3.47(\mathrm{~m}, 1 \mathrm{H}), 1.37-1.46(\mathrm{~m}, 2 \mathrm{H}), 0.91(\mathrm{~d}, J=7.83 \mathrm{~Hz}, 9 \mathrm{H}) ;{ }^{13} \mathrm{C} \mathrm{NMR}\left(\mathrm{CDCl}_{3}, 126\right.$ MHz) $\delta 163.7,163.5,162.8,162.7,156.1,151.9,133.1,129.6,129.5,129.1,129.0,115.6,115.4,66.7$, 


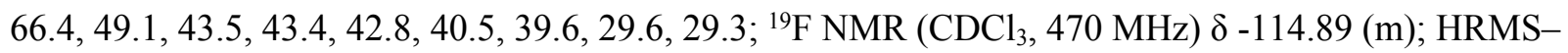
ESI (m/z): $[\mathrm{M}+\mathrm{H}]^{+}$calcd for, $\mathrm{C}_{21} \mathrm{H}_{27} \mathrm{FN}_{6} \mathrm{O}$; 399.2303; found, 399.2301

\section{4-((3-Fluoro-3-methylbutyl)(4-fluorobenzyl)amino)-6-morpholino-1,3,5-triazine-2-carbonitrile (54)} 4-((4-fluorobenzyl)amino)-6-morpholino-1,3,5-triazine-2-carbonitrile (100 mg, $0.318 \mathrm{mmol})$, sodium hydride (19.09 mg, $0.477 \mathrm{mmol}$ ), and 3-fluoro-3-methylbutyl 4-methylbenzenesulfonate ( $83 \mathrm{mg}, 0.318$ mmol) gave 4-((3-fluoro-3-methylbutyl)(4-fluorobenzyl)amino)-6-morpholino-1,3,5-triazine-2carbonitrile (59 mg, $0.147 \mathrm{mmol}, 46.1 \%$ yield); ${ }^{1} \mathrm{H} \mathrm{NMR}\left(\mathrm{CDCl}_{3}, 500 \mathrm{MHz}\right) \delta 7.17-7.25(\mathrm{~m}, 2 \mathrm{H}), 7.00$ $(\mathrm{t}, J=8.31 \mathrm{~Hz}, 2 \mathrm{H}), 4.76(\mathrm{~s}, 1 \mathrm{H}), 4.72(\mathrm{~s}, 1 \mathrm{H}), 3.75-3.87(\mathrm{~m}, 3 \mathrm{H}), 3.67-3.74(\mathrm{~m}, 4 \mathrm{H}), 3.63-3.67(\mathrm{~m}$, $1 \mathrm{H}), 3.60$ - $3.63(\mathrm{~m}, 1 \mathrm{H}), 3.54-3.60(\mathrm{~m}, 1 \mathrm{H}), 1.77-1.91(\mathrm{~m}, 2 \mathrm{H}), 1.37(\mathrm{~d}, J=18.10 \mathrm{~Hz}, 3 \mathrm{H}), 1.33(\mathrm{~d}, J=$ $18.10 \mathrm{~Hz}, 3 \mathrm{H}) ;{ }^{13} \mathrm{C} \mathrm{NMR}\left(\mathrm{CDCl}_{3}, 126 \mathrm{MHz}\right) \delta 164.1,163.9,163.6,163.4,163.2,163.1,161.2,161.1$, $152.0,151.9,132.9,129.6,129.5,129.1,115.5,115.4,115.3,95.1,94.8,93.8,93.4,66.6,66.3,49.3,49.2$ $43.9,43.7,43.4,42.5,42.0,41.9,38.3,38.2,37.6,37.4,26.8,26.7,26.6,26.5 ;{ }^{19} \mathrm{~F} \mathrm{NMR}\left(\mathrm{CDCl}_{3}, 470\right.$ MHz) $\delta$-114.71 (m), -140.96 (m), -139.84 (m); HRMS-ESI (m/z): $[\mathrm{M}+\mathrm{H}]^{+}$calcd for, $\mathrm{C}_{20} \mathrm{H}_{24} \mathrm{~F}_{2} \mathrm{~N}_{6} \mathrm{O}$; 403.2052; found, 403.2051

\section{4-((2-Cyclohexylethyl)(4-fluorobenzyl)amino)-6-morpholino-1,3,5-triazine-2-carbonitrile (55)}

General procedure B3 from 4-((4-fluorobenzyl)amino)-6-morpholino-1,3,5-triazine-2-carbonitrile (100 mg, $0.318 \mathrm{mmol}$ ), sodium hydride (19.09 mg, $0.477 \mathrm{mmol}$ ), and (2-bromoethyl)cyclohexane (122 mg, $0.636 \mathrm{mmol}$ ) gave 4-((2-cyclohexylethyl)(4-fluorobenzyl)amino)-6-morpholino-1,3,5-triazine-2carbonitrile (84.1 mg, $0.198 \mathrm{mmol}, 62.3 \%$ yield); ${ }^{1} \mathrm{H}$ NMR $\left(\mathrm{CDCl}_{3}, 500 \mathrm{MHz}\right) \delta 7.14-7.26(\mathrm{~m}, 2 \mathrm{H})$, $7.00(\mathrm{t}, J=8.56 \mathrm{~Hz}, 2 \mathrm{H}), 4.75(\mathrm{~s}, 1 \mathrm{H}), 4.71(\mathrm{~s}, 1 \mathrm{H}), 3.76-3.88(\mathrm{~m}, 3 \mathrm{H}), 3.60-3.76(\mathrm{~m}, 5 \mathrm{H}), 3.49-3.57$ (m, 1H), 3.39 - $3.49(\mathrm{~m}, 1 \mathrm{H}), 1.59$ - $1.74(\mathrm{~m}, 5 \mathrm{H}), 1.36$ - $1.48(\mathrm{~m}, 2 \mathrm{H}), 1.11$ - $1.24(\mathrm{~m}, 4 \mathrm{H}), 0.82$ - 0.98 $(\mathrm{m}, 2 \mathrm{H}) ;{ }^{13} \mathrm{C} \mathrm{NMR}\left(\mathrm{CDCl}_{3}, 126 \mathrm{MHz}\right) \delta 164.1,163.9,163.6,163.5,163.2,163.0,161.2,161.1,152.0$, $151.9,133.1,129.5,129.4,129.0,128.9,115.5,115.3,66.7,66.4,49.0,44.8,44.2,43.9,43.4,35.5,35.3$, 
34.7, 33.9, 33.2, 33.1, 26.4, 26.1, 14.1; HRMS-ESI (m/z): $[\mathrm{M}+\mathrm{H}]^{+}$calcd for, $\mathrm{C}_{23} \mathrm{H}_{29} \mathrm{FN} 6$ O; 425.2460; found, 425.2453

\section{4-((4-Fluorobenzyl)((3-methyloxetan-3-yl)methyl)amino)-6-morpholino-1,3,5-triazine-2-carbonitrile} (44)

In a dry reaction vial, 4-((4-fluorobenzyl)amino)-6-morpholino-1,3,5-triazine-2-carbonitrile (50 mg, $0.159 \mathrm{mmol})$ was dissolved into DMF (1 ml). Cesium carbonate $(104 \mathrm{mg}, 0.318 \mathrm{mmol})$ and 3(bromomethyl)-3-methyloxetane $(0.037 \mathrm{ml}, 0.318 \mathrm{mmol})$ were added to the solution and the reaction was left overnight at $80^{\circ} \mathrm{C}$. Crude was purified by FC (1-100\% EtOAc:Hexanes gradient) to afford 4-((4fluorobenzyl)((3-methyloxetan-3-yl)methyl)amino)-6-morpholino-1,3,5-triazine-2-carbonitrile (16.9 mg, $0.042 \mathrm{mmol}, 26.7 \%$ yield); ${ }^{1} \mathrm{H} \mathrm{NMR}\left(\mathrm{CDCl}_{3}, 500 \mathrm{MHz}\right) \delta 7.09-7.19(\mathrm{~m}, 2 \mathrm{H}), 7.01(\mathrm{t}, J=8.56 \mathrm{~Hz}, 2 \mathrm{H})$, $4.78(\mathrm{~s}, 1 \mathrm{H}), 4.71(\mathrm{~s}, 1 \mathrm{H}), 4.50-4.63(\mathrm{~m}, 2 \mathrm{H}), 4.36-4.42(\mathrm{~m}, 1 \mathrm{H}), 4.24-4.30(\mathrm{~m}, 2 \mathrm{H}), 3.82(\mathrm{dd}, J=$ 4.89, $9.78 \mathrm{~Hz}, 2 \mathrm{H}), 3.69$ - $3.79(\mathrm{~m}, 4 \mathrm{H}), 3.65(\mathrm{~d}, J=7.34 \mathrm{~Hz}, 2 \mathrm{H}), 2.93(\mathrm{~s}, 2 \mathrm{H}), 1.36(\mathrm{~s}, 3 \mathrm{H}) ;{ }^{13} \mathrm{C} \mathrm{NMR}$ $\left(\mathrm{CDCl}_{3}, 126 \mathrm{MHz}\right) \delta 165.1,163.5,155.5,152.0,151.9,132.3,129.2,129.1,128.7,115.7,115.6,115.5$, $115.3,115.2,81.5,81.3,79.5,79.3,72.4,69.3,66.6,66.3,52.7,51.5,50.3,50.2,43.9,43.5,43.4,41.0$, 40.7, 39.3, 39.2, 22.6, 22.1, 20.9; HRMS-ESI (m/z): $[\mathrm{M}+\mathrm{H}]^{+}$calcd for, $\mathrm{C}_{20} \mathrm{H}_{23} \mathrm{FN}_{6} \mathrm{O}_{2} ; 399.1939$; found, 399.1939

\section{4-((2-Aminoethyl)(4-fluorobenzyl)amino)-6-morpholino-1,3,5-triazine-2-carbonitrile (45)}

General Procedure B3 starting from 4-((4-fluorobenzyl)amino)-6-morpholino-1,3,5-triazine-2-carbonitrile (150 mg, $0.477 \mathrm{mmol}$ ) and tert-butyl (2-bromoethyl)carbamate (160 mg, $0.716 \mathrm{mmol})$ gave tert-butyl(2((4-cyano-6-morpholino-1,3,5-triazin-2-yl)(4-fluorobenzyl)amino) ethyl)carbamate (25.0 mg, 0.055

mmol, $11 \%$ yield). Carried directly through General Procedure B4 to give 4-((2-aminoethyl)(4fluorobenzyl)amino)-6-morpholino-1,3,5-triazine-2-carbonitrile (4.0 mg, $0.055 \mathrm{mmol}, 20.0 \%$ yield); ${ }^{1} \mathrm{H}$ NMR $\left(\mathrm{CDCl}_{3}, 500 \mathrm{MHz}\right) \delta 7.21(\mathrm{t}, \mathrm{J}=7.2 \mathrm{~Hz}, 2 \mathrm{H}), 7.01(\mathrm{t}, \mathrm{J}=8.3 \mathrm{~Hz}, 2 \mathrm{H}), 4.75(\mathrm{~s}, 2 \mathrm{H}), 3.86$ - 3.59 (m, 9H), $3.13(\mathrm{~s}, 2 \mathrm{H}), 1.33-1.17(\mathrm{~m}, 2 \mathrm{H}) .{ }^{13} \mathrm{C} \mathrm{NMR}\left(\mathrm{CDCl}_{3}, 126 \mathrm{MHz}\right) \delta 163.20,132.03,129.42,129.35$, 
115.92, 115.75, 66.53, 49.84, 44.12, 43.67, 14.20, 1.01. ${ }^{19} \mathrm{~F}$ NMR (470 MHz, Chloroform-d) $\delta$-75.70, 113.97. HRMS-ESI (m/z): $[\mathrm{M}+\mathrm{H}]^{+}$calcd for $\mathrm{C}_{17} \mathrm{H}_{20} \mathrm{FN}_{7} \mathrm{O}$, 358.1786; found, 358.1785

\section{4-((3-Aminopropyl)(4-fluorobenzyl)amino)-6-morpholino-1,3,5-triazine-2-carbonitrile (46)}

General Procedure B3 starting from 4-((4-fluorobenzyl)amino)-6-morpholino-1,3,5-triazine-2-carbonitrile (150 mg, $0.477 \mathrm{mmol}$ ) and tert-butyl (3-bromopropyl)carbamate (170 mg, $0.716 \mathrm{mmol})$ gave tert-butyl(3((4-cyano-6-morpholino-1,3,5-triazin-2-yl)(4-fluorobenzyl)amino) propyl)carbamate (97.6 mg, 0.207 mmol, 43.4 \% yield). Carried directly through General Proceure B4 to give 4-((3-aminopropyl)(4fluorobenzyl)amino)-6-morpholino-1,3,5-triazine-2-carbonitrile (19.0 mg, $0.085 \mathrm{mmol}, 60.0 \%$ yield); ${ }^{1} \mathrm{H}$ NMR $\left(\mathrm{CDCl}_{3}, 500 \mathrm{MHz}\right) \delta 7.23-7.09(\mathrm{~m}, 2 \mathrm{H}), 6.99(\mathrm{t}, \mathrm{J}=8.5 \mathrm{~Hz}, 2 \mathrm{H}), 4.82-4.64(\mathrm{~m}, 2 \mathrm{H}), 3.90-3.43$ (m, 9H), $2.93(\mathrm{~s}, 2 \mathrm{H}), 1.90(\mathrm{~s}, 3 \mathrm{H}), 1.26(\mathrm{~s}, 1 \mathrm{H}) .{ }^{13} \mathrm{C} \mathrm{NMR}\left(\mathrm{CDCl}_{3}, 126 \mathrm{MHz}\right) \delta 164.42,162.98,161.36$, $151.47,132.19,129.16,129.09,115.82,115.65,66.51,66.21,49.08,43.65,42.48,25.02 .{ }^{19} \mathrm{~F}$ NMR $(470$ MHz, Chloroform-d) $\delta-74.98,-76.05,-113.87--115.03(\mathrm{~m})$. HRMS-ESI (m/z): $[\mathrm{M}+\mathrm{H}]^{+}$calcd for $\mathrm{C}_{18} \mathrm{H}_{22} \mathrm{FN}_{7} \mathrm{O}$, 372.1943; found, 372.1943

\section{4-((4-Aminobutyl)(4-fluorobenzyl)amino)-6-morpholino-1,3,5-triazine-2-carbonitrile (47)}

General Procedure B3 starting from 4-((4-fluorobenzyl)amino)-6-morpholino-1,3,5-triazine-2-carbonitrile (150 mg, $0.477 \mathrm{mmol}$ ) and tert-butyl (4-bromobutyl)carbamate (180 mg, $0.716 \mathrm{mmol})$ gave tert-butyl(4((4-cyano-6-morpholino-1,3,5-triazin-2-yl)(4-fluorobenzyl)amino) butyl)carbamate (40 mg, $0.082 \mathrm{mmol}$, $17 \%$ yield). Carried directly through General Procedure B4 to give 4-((4-aminobutyl)(4fluorobenzyl)amino)-6-morpholino-1,3,5-triazine-2-carbonitrile (4.9 mg, $0.013 \mathrm{mmol}, 15 \%$ yield); ${ }^{1} \mathrm{H}$ $\operatorname{NMR}\left(\mathrm{CDCl}_{3}, 500 \mathrm{MHz}\right) \delta 7.19(\mathrm{~d}, \mathrm{~J}=6.3 \mathrm{~Hz}, 2 \mathrm{H}), 7.00(\mathrm{~d}, \mathrm{~J}=4.4 \mathrm{~Hz}, 2 \mathrm{H}), 4.73(\mathrm{~d}, \mathrm{~J}=15.5 \mathrm{~Hz}, 2 \mathrm{H})$, $3.91-3.58(\mathrm{~m}, 8 \mathrm{H}), 3.50(\mathrm{~s}, 2 \mathrm{H}), 2.95(\mathrm{~d}, \mathrm{~J}=54.8 \mathrm{~Hz}, 2 \mathrm{H}), 1.62(\mathrm{~s}, 4 \mathrm{H}) .{ }^{13} \mathrm{C} \mathrm{NMR}\left(\mathrm{CDCl}_{3}, 126 \mathrm{MHz}\right) \delta$ 164.20, 163.32, 151.81, 129.50, 129.08, 129.01, 115.68, 115.51, 49.27, 45.42, 39.34, 24.04. ${ }^{19} \mathrm{~F}$ NMR 
(470 MHz, Chloroform-d) $\delta$-75.87, -114.63. HRMS-ESI (m/z): $[\mathrm{M}+\mathrm{H}]^{+}$calcd for $\mathrm{C}_{19} \mathrm{H}_{24} \mathrm{FN}_{7} \mathrm{O}$, 386.2099; found, 386.2095

4-((4-Fluorobenzyl)(2-(pyrrolidin-1-yl)ethyl)amino)-6-morpholino-1,3,5-triazine-2-carbonitrile (48) General procedure B3 from 4-((4-fluorobenzyl)amino)-6-morpholino-1,3,5-triazine-2-carbonitrile (100 $\mathrm{mg}, 0.318 \mathrm{mmol})$, sodium hydride $(19.09 \mathrm{mg}, 0.477 \mathrm{mmol})$, and 1-(2-chloroethyl)pyrrolidine (85 mg, $0.636 \mathrm{mmol})$ gave 4-((4-fluorobenzyl)(2-(pyrrolidin-1-yl)ethyl)amino)-6-morpholino-1,3,5-triazine-2carbonitrile (92 mg, $0.224 \mathrm{mmol}, 70.3 \%$ yield); ${ }^{1} \mathrm{H} \mathrm{NMR}\left(\mathrm{CDCl}_{3}, 500 \mathrm{MHz}\right) \delta 7.15$ - $7.25(\mathrm{~m}, 2 \mathrm{H}), 6.94$ 7.05 (m, 2H), 4.80 (s, 1H), 4.78 (s, 1H), 3.81 (br. s., 1H), 3.78 (br. s., $2 \mathrm{H}), 3.68$ - 3.74 (m, 3H), $3.62-3.68$ (m, 3H), $3.58(\mathrm{t}, J=7.34 \mathrm{~Hz}, 1 \mathrm{H}), 2.56-2.67(\mathrm{~m}, 2 \mathrm{H}), 2.42-2.56(\mathrm{~m}, 4 \mathrm{H}), 1.76$ (br. s., $4 \mathrm{H}) ;{ }^{13} \mathrm{C} \mathrm{NMR}$ $\left(\mathrm{CDCl}_{3}, 126 \mathrm{MHz}\right) \delta 164.2,164.1,163.6,163.5,163.2,163.0,161.2,161.1,152.0,151.9,133.1,133.0$ $129.6,129.5,129.0,128.9,115.5,115.3,66.6,66.4,66.3,54.4,54.3,53.6,52.7,49.8,49.7,45.7,45.4$, 43.9, 43.4, 23.5, 23.4; HRMS-ESI (m/z): $[\mathrm{M}+\mathrm{H}]^{+}$calcd for, $\mathrm{C}_{21} \mathrm{H}_{26} \mathrm{FN}_{7} \mathrm{O}$; 412.2256; found, 412.2255

4-((4-Fluorobenzyl)(2-(piperidin-3-yl)ethyl)amino)-6-morpholino-1,3,5-triazine-2-carbonitrile (60)

General procedure B4 from tert-butyl 3-(2-((4-cyano-6-morpholino-1,3,5-triazin-2-yl)(4fluorobenzyl)amino)ethyl)piperidine-1-carboxylate $(80 \mathrm{mg}, 0.152 \mathrm{mmol})$ gave 4-((4-fluorobenzyl)(2(piperidin-3-yl)ethyl)amino)-6-morpholino-1,3,5-triazine-2-carbonitrile (45.6 mg, 0.107 mmol, $70.4 \%$ yield); ${ }^{1} \mathrm{H}$ NMR $\left(\mathrm{CDCl}_{3}, 500 \mathrm{MHz}\right) \delta 7.12$ - 7.24 (m, 2H), 6.92 - 7.09 (m, 2H), 4.57 - 4.86 (m, 2H), 3.53 $3.99(\mathrm{~m}, 10 \mathrm{H}), 3.31$ - 3.52 (m, 2H), 2.84 (br. s., 1H), 2.56 (br. s., 1H), 1.85 - 2.02 (m, 2H), 1.78 (br. s., 2H), 1.35 - $1.62(\mathrm{~m}, 2 \mathrm{H}), 1.03$ - $1.26(\mathrm{~m}, 1 \mathrm{H}) ;{ }^{13} \mathrm{C} \mathrm{NMR}\left(\mathrm{CDCl}_{3}, 126 \mathrm{MHz}\right) \delta 169.9,168.6,164.4,164.1$, 164.0, 163.6, 163.4, 161.3, 161.0, 160.8, 151.9, 129.5, 129.0, 115.7, 115.5, 107.8, 66.6, 66.3, 49.0, 44.7, 44.0, 43.6, 43.4, 31.6, 31.5, 30.8, 30.5, 28.9, 28.8, 22.0; ${ }^{19} \mathrm{~F} \mathrm{NMR}\left(\mathrm{CDCl}_{3}, 470 \mathrm{MHz}\right) \delta-114.80(\mathrm{~s})$, 75.85 (s); HRMS-ESI (m/z): $[\mathrm{M}+\mathrm{H}]^{+}$calcd for, $\mathrm{C}_{22} \mathrm{H}_{28} \mathrm{FN}_{7} \mathrm{O}$; 426.2412 ; found, 426.2398 
General procedure B4 from tert-butyl 4-(2-((4-cyano-6-morpholino-1,3,5-triazin-2-yl)(4-

fluorobenzyl)amino)ethyl)piperidine-1-carboxylate $(80 \mathrm{mg}, 0.152 \mathrm{mmol})$ gave 4-((4-fluorobenzyl)(2(piperidin-4-yl)ethyl)amino)-6-morpholino-1,3,5-triazine-2-carbonitrile (12 mg, $0.028 \mathrm{mmol}, 18.53 \%$ yield); ${ }^{1} \mathrm{H} \mathrm{NMR}\left(\mathrm{CDCl}_{3}, 500 \mathrm{MHz}\right) \delta 7.15-7.24(\mathrm{~m}, 2 \mathrm{H}), 7.02(\mathrm{t}, J=8.31 \mathrm{~Hz}, 2 \mathrm{H}), 4.77(\mathrm{~s}, 1 \mathrm{H}), 4.72(\mathrm{~s}$, 1H), 3.84 (br. s, 2H), $3.70-3.78(\mathrm{~m}, 4 \mathrm{H}), 3.63-3.70(\mathrm{~m}, 2 \mathrm{H}), 3.56(\mathrm{t}, J=7.09 \mathrm{~Hz}, 1 \mathrm{H}), 3.44-3.52(\mathrm{~m}$, 1H), 3.24 - $3.34(\mathrm{~m}, 2 \mathrm{H}), 2.69-2.86(\mathrm{~m}, 2 \mathrm{H}), 1.73$ - $1.92(\mathrm{~m}, 2 \mathrm{H}), 1.39-1.58(\mathrm{~m}, 5 \mathrm{H}) ;{ }^{19} \mathrm{~F} \mathrm{NMR}\left(\mathrm{CDCl}_{3}\right.$, $470 \mathrm{MHz}) \delta$-114.58 (m) -75.84 (m); HRMS-ESI (m/z): [M+H] ${ }^{+}$calcd for, $\mathrm{C}_{22} \mathrm{H}_{28} \mathrm{FN}_{7} \mathrm{O} ; 426.2412$; found, 426.2408

\section{4-((4-Fluorobenzyl)(piperidin-3-ylmethyl)amino)-6-morpholino-1,3,5-triazine-2-carbonitrile (62)}

General procedure B4 from tert-butyl 3-(((4-cyano-6-morpholino-1,3,5-triazin-2-yl)(4fluorobenzyl)amino)methyl) piperidine-1-carboxylate (80 mg, $0.156 \mathrm{mmol})$ gave 4-((4fluorobenzyl)(piperidin-3-ylmethyl)amino)-6-morpholino-1,3,5-triazine-2-carbonitrile, TFA (47 mg, $0.089 \mathrm{mmol}, 57.2 \%$ yield). ${ }^{1} \mathrm{H}$ NMR $\left(\mathrm{CDCl}_{3}, 500 \mathrm{MHz}\right) \delta 7.10-7.24(\mathrm{~m}, 2 \mathrm{H}), 7.00(\mathrm{t}, J=7.83 \mathrm{~Hz}, 2 \mathrm{H})$, 4.84 - $4.99(\mathrm{~m}, 1 \mathrm{H}), 4.49$ - $4.68(\mathrm{~m}, 1 \mathrm{H}), 3.60$ - $3.93(\mathrm{~m}, 9 \mathrm{H}), 3.22$ - $3.54(\mathrm{~m}, 4 \mathrm{H}), 2.81$ - $3.02(\mathrm{~m}, 1 \mathrm{H})$, $2.57-2.81$ (m, 1H), 2.29 (br. s., $1 \mathrm{H}), 1.93$ (br. s., $1 \mathrm{H}), 1.70-1.87$ (m, 2H), $1.14-1.40$ (m, 1H); ${ }^{13} \mathrm{C}$ NMR $\left(\mathrm{CDCl}_{3}, 126 \mathrm{MHz}\right) \delta 164.9,164.8,163.4,163.3,163.2,161.3,152.0,151.8,132.2,132.1,129.5,129.1$, $129.0,115.8,115.5,115.3,115.2,66.5,66.2,49.5,49.1,48.3,47.7,47.2,44.6,44.5,43.9,43.5,43.3$, 32.5, 32.4, 28.1, 26.8, 26.3, 21.8, 21.3; ${ }^{19} \mathrm{~F} \mathrm{NMR}\left(\mathrm{CDCl}_{3}, 470 \mathrm{MHz}\right) \delta$-75.94, -114.44; HRMS-ESI (m/z): $[\mathrm{M}+\mathrm{H}]^{+}$calcd for, $\mathrm{C}_{21} \mathrm{H}_{26} \mathrm{FN}_{7} \mathrm{O} ; 412.2256$; found, 412.2255

\section{4-((4-Fluorobenzyl)(piperidin-4-ylmethyl)amino)-6-morpholino-1,3,5-triazine-2-carbonitrile (63)}

General procedure B4 from tert-butyl 4-(((4-cyano-6-morpholino-1,3,5-triazin-2-yl)(4-

fluorobenzyl)amino)methyl)piperidine-1-carboxylate (33 mg, $0.065 \mathrm{mmol})$ gave 4-((4fluorobenzyl)(piperidin-4-ylmethyl)amino)-6-morpholino-1,3,5-triazine-2-carbonitrile, TFA (23.5 mg, $0.045 \mathrm{mmol}, 69.3 \%$ yield). ${ }^{1} \mathrm{H}$ NMR $\left(\mathrm{CDCl}_{3}, 500 \mathrm{MHz}\right) \delta 7.12-7.24(\mathrm{~m}, 2 \mathrm{H}), 7.01(\mathrm{t}, J=8.31 \mathrm{~Hz}, 2 \mathrm{H})$, 
4.79 (s, 1H), 4.74 (s, 1H), 3.82 (br. s., 2H), 3.72 (br. s., 4H), 3.67 (br. s., 2H), 3.34 - 3.53 (m, 4H), 2.86 (br. s., 2H), 1.94 - $2.16(\mathrm{~m}, 1 \mathrm{H}), 1.72-1.90$ (m, 2H), 1.57 (br. s., $2 \mathrm{H}) ;{ }^{13} \mathrm{C} \mathrm{NMR}\left(\mathrm{CDCl}_{3}, 126 \mathrm{MHz}\right) \delta$ $165.0,164.8,163.5,163.4,161.4,161.3,152.0,132.4,129.5,129.4,129.0,128.9,115.8,115.7,115.6$, $115.3,66.6,66.3,51.5,50.6,50.4,49.8,44.0,43.9,43.5,43.4,32.9,32.8,26.6,26.4 ;{ }^{19} \mathrm{~F} \mathrm{NMR}\left(\mathrm{CDCl}_{3}\right.$, $470 \mathrm{MHz}) \delta$-75.82(m), -114.32 (m); HRMS-ESI (m/z): $[\mathrm{M}+\mathrm{H}]^{+}$calcd for, $\mathrm{C}_{21} \mathrm{H}_{26} \mathrm{FN}_{7} \mathrm{O} ; 412.2256$; found, 412.2257

\section{4-((4-Fluorobenzyl)((tetrahydro-2H-pyran-4-yl)methyl)amino)-6-morpholino-1,3,5-triazine-2- carbonitrile (64)}

General procedure B3 from 4-((4-fluorobenzyl)amino)-6-morpholino-1,3,5-triazine-2-carbonitrile (100 $\mathrm{mg}, 0.318 \mathrm{mmol})$, sodium hydride $(15.27 \mathrm{mg}, 0.382 \mathrm{mmol}$ ) and 4-(bromomethyl)tetrahydro-2H-pyran (0.042 ml, $0.318 \mathrm{mmol})$ gave 4-((4-fluorobenzyl)((tetrahydro-2H-pyran-4-yl)methyl)amino)-6-

morpholino-1,3,5-triazine-2-carbonitrile (68 mg, 0.165 mmol, $51.8 \%$ yield); ${ }^{1} \mathrm{H}$ NMR $\left(\mathrm{CDCl}_{3}, 500 \mathrm{MHz}\right)$ $\delta 7.11-7.23(\mathrm{~m}, 2 \mathrm{H}), 6.99(\mathrm{t}, J=8.31 \mathrm{~Hz}, 2 \mathrm{H}), 4.79(\mathrm{~s}, 1 \mathrm{H}), 4.74(\mathrm{~s}, 1 \mathrm{H}), 3.89-4.02(\mathrm{~m}, 2 \mathrm{H}), 3.58-3.86$ (m, 9H), $3.43(\mathrm{~d}, J=7.34 \mathrm{~Hz}, 1 \mathrm{H}), 3.37$ (d, $J=7.34 \mathrm{~Hz}, 1 \mathrm{H}), 3.26-3.35(\mathrm{~m}, 2 \mathrm{H}), 1.93-2.02(\mathrm{~m}, 1 \mathrm{H})$, 1.46 - $1.57(\mathrm{~m}, 2 \mathrm{H}), 1.27$ - $1.41(\mathrm{~m}, 2 \mathrm{H}) ;{ }^{13} \mathrm{C} \mathrm{NMR}\left(\mathrm{CDCl}_{3}, 126 \mathrm{MHz}\right) \delta 164.8,164.7,163.5,163.2$, $163.1,161.3,161.1,152.0,132.9,132.8,129.4,128.9,128.8,115.6,115.5,115.4,77.3,77.1,76.8,67.6$, $66.6,66.4,66.3,52.4,51.5,50.2,49.8,44.0,43.4,34.0,33.8,31.0,30.6{ }^{19} \mathrm{~F} \mathrm{NMR}\left(\mathrm{CDCl}_{3}, 470 \mathrm{MHz}\right) \delta$ 114.74 (m); HRMS-ESI (m/z): [M+H] $]^{+}$calcd for, $\mathrm{C}_{21} \mathrm{H}_{25} \mathrm{FN}_{6} \mathrm{O}_{2} ; 413.2096$; found, 413.2098

\section{4-((4-Fluorobenzyl)(pyrrolidin-3-ylmethyl)amino)-6-morpholino-1,3,5-triazine-2-carbonitrile (66)}

General procedure B4 from tert-butyl 3-(((4-cyano-6-morpholino-1,3,5-triazin-2-yl)(4-

fluorobenzyl)amino)methyl)pyrrolidine-1-carboxylate (30 mg, $0.060 \mathrm{mmol})$ gave 4-((4-

fluorobenzyl)(pyrrolidin-3-ylmethyl)amino)-6-morpholino-1,3,5-triazine-2-carbonitrile, TFA (23 mg, $0.045 \mathrm{mmol}, 74.6 \%$ yield); ${ }^{1} \mathrm{H} \mathrm{NMR}\left(\mathrm{CDCl}_{3}, 500 \mathrm{MHz}\right) \delta 7.12-7.24(\mathrm{~m}, 2 \mathrm{H}), 7.00(\mathrm{t}, J=8.31 \mathrm{~Hz}, 2 \mathrm{H})$, 4.64 - 4.85 (m, 2H), 3.77 - 3.88 (m, 2H), 3.53 - 3.75 (m, 8H), 3.45 (br. s., 1H), 3.32 (br. s., 2H), 2.94 - 
$3.13(\mathrm{~m}, 1 \mathrm{H}), 2.65-2.84(\mathrm{~m}, 1 \mathrm{H}), 1.99-2.15(\mathrm{~m}, 1 \mathrm{H}), 1.67-1.82(\mathrm{~m}, J=7.60,12.00 \mathrm{~Hz}, 1 \mathrm{H}) ;{ }^{13} \mathrm{C} \mathrm{NMR}$ $\left(\mathrm{CDCl}_{3}, 126 \mathrm{MHz}\right) \delta 164.8,163.5,163.3,161.4,161.3,152.0,151.8,132.4,132.3,129.5,129.0,115.8$ $115.6,115.3,115.2,66.6,66.3,49.8,48.6,48.0,47.4,45.2,45.0,44.0,43.5,43.4,37.3,37.0,28.5,28.1$; ${ }^{19} \mathrm{~F}$ NMR $\left(\mathrm{CDCl}_{3}, 470 \mathrm{MHz}\right) \delta-75.83(\mathrm{~m}),-114.24(\mathrm{~m})$; HRMS-ESI $(\mathrm{m} / \mathrm{z}):[\mathrm{M}+\mathrm{H}]^{+}$calcd for, $\mathrm{C}_{20} \mathrm{H}_{24} \mathrm{FN}_{7} \mathrm{O}$; 398.2099; found, 398.2102

\section{4-((4-fluorobenzyl)((tetrahydro-2H-pyran-3-yl)methyl)amino)-6-morpholino-1,3,5-triazine-2- carbonitrile (65)}

General procedure B3 from 4-((4-fluorobenzyl)amino)-6-morpholino-1,3,5-triazine-2-carbonitrile (100 mg, $0.318 \mathrm{mmol})$, sodium hydride $(15.27 \mathrm{mg}, 0.382 \mathrm{mmol})$, and (tetrahydro-2H-pyran-3-yl)methyl 4methylbenzenesulfonate (86 mg, $0.318 \mathrm{mmol})$ gave 4-((4-fluorobenzyl)((tetrahydro-2H-pyran-3yl)methyl)amino)-6-morpholino-1,3,5-triazine-2-carbonitrile (57 mg, $0.138 \mathrm{mmol}, 43.4 \%$ yield); ${ }^{1} \mathrm{H}$ $\operatorname{NMR}\left(\mathrm{CDCl}_{3}, 500 \mathrm{MHz}\right) \delta 7.52$ - $7.58(\mathrm{~m}, 1 \mathrm{H}), 7.43$ - $7.52(\mathrm{~m}, 2 \mathrm{H}), 7.36-7.43(\mathrm{~m}, 1 \mathrm{H}), 4.85(\mathrm{~s}, 1 \mathrm{H})$, $4.78(\mathrm{~s}, 1 \mathrm{H}), 3.82$ - $3.88(\mathrm{~m}, 1 \mathrm{H}), 3.77$ - $3.82(\mathrm{~m}, 2 \mathrm{H}), 3.71$ - $3.77(\mathrm{~m}, 2 \mathrm{H}), 3.68$ (br. s., $1 \mathrm{H}), 3.56$ - 3.66 $(\mathrm{m}, 3 \mathrm{H}), 3.45-3.51(\mathrm{~m}, 1 \mathrm{H}), 1.50-1.61(\mathrm{~m}, 1 \mathrm{H}), 1.37-1.50(\mathrm{~m}, 2 \mathrm{H}), 0.92(\mathrm{~d}, J=6.36 \mathrm{~Hz}, 3 \mathrm{H}), 0.90(\mathrm{~d}$, $J=6.36 \mathrm{~Hz}, 3 \mathrm{H}) ;{ }^{13} \mathrm{C} \mathrm{NMR}\left(\mathrm{CDCl}_{3}, 126 \mathrm{MHz}\right) \delta 164.2,164.1,163.6,163.4,152.0,151.9,138.7,138.4$, $131.0,130.7,130.4,129.1,124.3,124.2,124.1,115.5,115.4,66.6,66.3,66.2,49.7,49.5,45.8,44.9,43.9$ 43.4, 36.3, 35.4, 26.0, 25.8, 22.5, 22.4; HRMS-ESI (m/z): $[\mathrm{M}+\mathrm{H}]^{+}$calcd for, $\mathrm{C}_{21} \mathrm{H}_{25} \mathrm{FN}_{6} \mathrm{O}_{2} ; 413.2096$; found, 413.2149

\section{4-morpholino-6-((2-nitrobenzyl)(piperidin-3-ylmethyl)amino)-1,3,5-triazine-2-carbonitrile (67)}

General procedure B4 from tert-butyl 3-(((4-cyano-6-morpholino-1,3,5-triazin-2-yl)(2-

nitrobenzyl)amino)methyl)piperidine-1-carboxylate (40 mg, 0.074 mmol) gave 4-morpholino-6-((2nitrobenzyl)(piperidin-3-ylmethyl)amino)-1,3,5-triazine-2-carbonitrile, TFA (25 mg, 0.045 mmol, $60.9 \%$ yield); ${ }^{1} \mathrm{H} \mathrm{NMR}\left(\mathrm{CDCl}_{3}, 500 \mathrm{MHz}\right) \delta 8.03-8.12(\mathrm{~m}, 1 \mathrm{H}), 7.58(\mathrm{t}, J=7.34 \mathrm{~Hz}, 1 \mathrm{H}), 7.46(\mathrm{t}, J=6.85 \mathrm{~Hz}$, 1H), 7.15 (br. s., 1H), 5.14 - 5.30 (m, 1H), 4.93 - 5.13 (m, 1H), 3.86 (br. s., $1 \mathrm{H}), 3.78$ (d, $J=18.59 \mathrm{~Hz}$, 
4H), 3.67 (br. s., 1H), 3.56 (br. s., 1H), 3.34 - 3.51 (m, 3H), 3.03 (br. s., 1H), 2.89 (br. s., 1H), 2.28 - 2.50 (m, 1H), $1.94-2.06(\mathrm{~m}, 1 \mathrm{H}), 1.74-1.94(\mathrm{~m}, 2 \mathrm{H}), 1.35-1.49(\mathrm{~m}, 1 \mathrm{H}), 1.16-1.35(\mathrm{~m}, 2 \mathrm{H}) ;{ }^{13} \mathrm{C} \mathrm{NMR}$ $\left(\mathrm{CDCl}_{3}, 126 \mathrm{MHz}\right) \delta 165.3,165.0,163.4,163.2,152.0,151.8,148.4,133.9,133.8,132.5,132.2,128.5$, $128.4,128.1,127.7,125.5,125.3,115.0,66.5,66.1,49.5,49.4,48.1,47.9,47.2,44.9,44.7,43.9,43.3$, 33.0, 32.7, 26.8, 26.2, 21.8, 21.2, 17.7; ${ }^{19} \mathrm{~F} \mathrm{NMR}\left(\mathrm{CDCl}_{3}, 470 \mathrm{MHz}\right) \delta-75.82 ; \mathrm{HRMS}-\mathrm{ESI}(\mathrm{m} / \mathrm{z}):[\mathrm{M}+\mathrm{H}]^{+}$ calcd for, $\mathrm{C}_{21} \mathrm{H}_{26} \mathrm{~N}_{8} \mathrm{O}_{3} ; 439.2201$; found, 439.2208

\section{4-(((5,5-difluoropiperidin-3-yl)methyl)(4-fluorobenzyl)amino)-6-morpholino-1,3,5-triazine-2- carbonitrile, TFA (68)}

General procedure B4 from tert-butyl 5-(((4-cyano-6-morpholino-1,3,5-triazin-2-yl)(4-

fluorobenzyl)amino)methyl)-3,3-difluoropiperidine-1-carboxylate (31.6 mg, $0.058 \mathrm{mmol})$ gave 4-(((5,5difluoropiperidin-3-yl)methyl)(4-fluorobenzyl)amino)-6-morpholino-1,3,5-triazine-2-carbonitrile, TFA (29 mg, $0.052 \mathrm{mmol}, 89 \%$ yield); ${ }^{1} \mathrm{H}$ NMR $\left(\mathrm{CDCl}_{3}, 500 \mathrm{MHz}\right) \delta 7.13-7.23(\mathrm{~m}, 2 \mathrm{H}), 7.02$ (t, $J=8.31$ Hz, 2H), 4.83 - $4.97(\mathrm{~m}, 1 \mathrm{H}), 4.55$ - $4.75(\mathrm{~m}, 1 \mathrm{H}), 3.77$ - $3.94(\mathrm{~m}, 2 \mathrm{H}), 3.65$ - 3.77 (m, 7H), 3.60 (br. s., 1H), $3.40-3.55(\mathrm{~m}, 1 \mathrm{H}), 3.34(\mathrm{~d}, J=12.23 \mathrm{~Hz}, 1 \mathrm{H}), 3.07-3.26(\mathrm{~m}, 1 \mathrm{H}), 2.85(\mathrm{t}, J=11.98 \mathrm{~Hz}, 1 \mathrm{H}), 2.66$ - 2.78 (m, 1H), 2.59 (br. s., $1 \mathrm{H}), 2.25$ (br. s., $1 \mathrm{H}), 1.67$ - $1.91(\mathrm{~m}, 1 \mathrm{H}) ;{ }^{13} \mathrm{C} \mathrm{NMR}\left(\mathrm{CDCl}_{3}, 126 \mathrm{MHz}\right) \delta$ $165.1,165.0,163.5,163.3,152.1,151.9,131.9,129.7,129.6,129.2,129.1,115.9,115.9,115.8,115.7$,

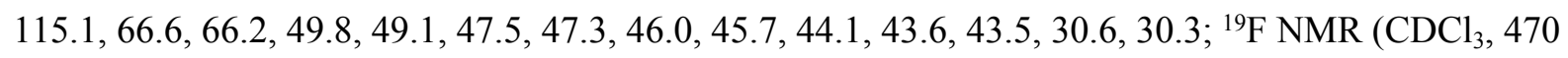
MHz) $\delta$ ppm -113.98 (s) -101.29 (s) -100.74 (s) -100.27 (s) -99.79 (s) -99.25 (s) -98.70 (s) -75.80 (s); HRMS-ESI (m/z): $[\mathrm{M}+\mathrm{H}]^{+}$calcd for, $\mathrm{C}_{19} \mathrm{H}_{31} \mathrm{~N}_{7} \mathrm{O}$; 448.2069 ; found, 448.2069 